TRANSACTIONS OF THE

AMERICAN MATHEMATICAL SOCIETY

Volume 351, Number 2, February 1999, Pages 683-713

S 0002-9947(99)02021-8

\title{
THE HEAT KERNEL WEIGHTED HODGE LAPLACIAN ON NONCOMPACT MANIFOLDS
}

\author{
EDWARD L. BUELER
}

\begin{abstract}
On a compact orientable Riemannian manifold, the Hodge Laplacian $\triangle$ has compact resolvent, therefore a spectral gap, and the dimension of the space $\mathcal{H}^{p}=\operatorname{ker} \triangle^{p}$ of harmonic $p$-forms is a topological invariant. By contrast, on complete noncompact Riemannian manifolds, $\triangle$ is known to have various pathologies, among them the absence of a spectral gap and either "too large" or "too small" a space $\mathcal{H}^{p}$.

In this article we use a heat kernel measure $d \mu$ to determine the space of square-integrable forms and to construct the appropriate Laplacian $\triangle_{\mu}$. We recover in the noncompact case certain results of Hodge's theory of $\Delta$ in the compact case. If the Ricci curvature of a noncompact connected Riemannian manifold $M$ is bounded below, then this "heat kernel weighted Laplacian" $\triangle_{\mu}$ acts on functions on $M$ in precisely the manner we would wish, that is, it has a spectral gap and a one-dimensional kernel. We prove that the kernel of $\triangle_{\mu}$ on $n$-forms is zero-dimensional on $M$, as we expect from topology, if the Ricci curvature is nonnegative. On Euclidean space, there is a complete Hodge theory for $\triangle_{\mu}$. Weighted Laplacians also have a duality analogous to Poincaré duality on noncompact manifolds. Finally, we show that heat kernel-like measures give desirable spectral properties (compact resolvent) in certain general cases. In particular, we use measures with Gaussian decay to justify the statement that every topologically tame manifold has a strong Hodge decomposition.
\end{abstract}

\section{INTRODUCTION}

The Hodge-de Rham theorem for a compact Riemannian manifold $M$ relates the geometrically-defined spaces of harmonic differential forms on $M$ to the topologically-invariant cohomology groups. A self-contained treatment of the theorem is given in [Wa]. Extensions of this simple relation to noncompact manifolds have been an area of active research for several decades. See, for example, [Ga], [AV], [deR], [Dod], and [EF2].

In this article we will develop an extension of Hodge theory to complete orientable Riemannian manifolds $M$ which uses a heat kernel measure as a weight in determining the size of the space of square integrable forms and to determine the relevant Laplacian operator. We will define this operator in Section 2, so for now we merely outline it.

Let $\left(M^{n}, g\right)$ be connected, oriented, and complete. Choose $t>0$ and $x_{0} \in$ $M$, and let $x \mapsto \rho_{t}\left(x_{0}, x\right)$ be the associated heat kernel. By definition, $\rho$ is the

Received by the editors July 29, 1996 and, in revised form, February 5, 1997.

1991 Mathematics Subject Classification. Primary 58A14, 35J10, 58G11.

Key words and phrases. Hodge theory, heat kernels, weighted cohomology, Schrödinger operators. 
fundamental solution of the ordinary heat equation $\frac{\partial u}{\partial t}=-\Delta u$ on functions. The heat kernel $\rho$ is unique if we assume that the Ricci curvature of $M$ is bounded below. Define

$$
d \mu=\rho_{t}\left(x_{0}, x\right) d x,
$$

a heat kernel measure on $M$, where $d x$ is the Riemann-Lebesgue measure determined by $g$. Consider those differential forms which are square integrable with respect to $d \mu$-that is, consider the Hilbert space $L_{\mu}^{2} \Omega^{\bullet}$. Let $d_{\mu}^{*}$ be the adjoint of the exterior derivative $d$ on $L_{\mu}^{2} \Omega^{\bullet}$. The resulting "heat kernel weighted Hodge Laplacian"

$$
\triangle_{\mu}=d_{\mu}^{*} d+d d_{\mu}^{*}
$$

is a nonnegative, self-adjoint operator. Let $\mathcal{H}_{\mu}^{p}=\operatorname{ker} \triangle_{\mu}^{p}$.

1.1 Conjecture. Let $\left(M^{n}, g\right)$ be connected, oriented, and complete. Suppose $\mathrm{Ric}_{M}$ $\geq-c$ for some $c>0$. Fix $t>0$ and $x_{0} \in M$. Let $d \mu$ be the heat kernel measure $\bar{d} \mu=\rho_{t}\left(x_{0}, x\right) d x$. Then $\triangle_{\mu}$ has a spectral gap and for $0 \leq p \leq n$,

$$
\mathcal{H}_{\mu}^{p} \cong H_{\text {de Rham }}^{p}(M) .
$$

Why might $\triangle_{\mu}$ satisfy a Hodge-de Rham theorem for such a class of Riemannian manifolds? Our evidence is as follows.

The conjecture is correct if $p=0$, that is, for $\triangle_{\mu}^{0}$ acting on functions in $L_{\mu}^{2} \Omega^{0}$. Specifically, Theorem 6.1 says ker $\triangle_{\mu}^{0}=$ \{constants $\}$ and $\triangle_{\mu}^{0}$ has a spectral gap (of size $\frac{1}{2 t}$ for small $t$ ). This result extends to complete manifolds a theorem of $\mathrm{E}$. Hsu, B. Driver, and Y. Hu ([Hs] and $[\mathrm{DriH}])$.

It is reasonable to assume that for a large class of manifolds, the inverse of the heat kernel is not integrable if $M$ is not compact. For such manifolds, ker $\triangle_{\mu}^{n}=0$ if $M$ is not compact. In fact, there are nonvanishing harmonic $n$-forms under $\triangle_{\mu}$ iff $\frac{1}{\rho_{t}\left(x_{0}, x\right)}$ is integrable (Theorem 6.2). If the Ricci curvature of $M$ is nonnegative, for instance, then $\mathcal{H}_{\mu}^{n} \cong H_{\text {de Rham }}^{n}(M)$.

On Euclidean space of dimension $n$ our construction yields an apparently unnoticed "Hodge theorem of $R^{n}$ " (Theorem 7.1). Indeed, on $R^{n}$ with the usual metric, we have

$$
\mathcal{H}_{\mu}^{p}=\left\{\begin{array}{ll}
\{\text { constants }\}, & p=0 \\
0, & p>0
\end{array} \cong H_{\text {de Rham }}^{p}\left(R^{n}\right)\right.
$$

Furthermore, $\triangle_{\mu}$ has compact resolvent. The weighted Laplacian $\triangle_{\mu^{-}}$corresponding to the measure

$$
d \mu^{-}=\frac{1}{\rho_{t}\left(x_{0}, x\right)} d x
$$

also has compact resolvent on $R^{n}$, and harmonic forms under $\triangle_{\mu^{-}}$bear a topological interpretation

$$
\mathcal{H}_{\mu^{-}}^{p} \cong H_{\mathrm{de} \mathrm{R,c}}^{p}\left(R^{n}\right)
$$

(where the right-hand side is the compactly-supported de Rham cohomology of $\left.R^{n}\right)$. These facts are in accordance with Poincaré duality on $R^{n}$.

Let us consider more general $d \mu$ on $M$ : suppose $d \mu=e^{2 h} d x$ for some $h \in$ $C^{\infty}(M)$. Each such $d \mu$ yields a weighted Hodge Laplacian $\triangle_{\mu}$ which acts in $L_{\mu}^{2} \Omega^{\bullet}$. 
Any such weighted Laplacian $\triangle_{\mu}$ gives the same decomposition theorem on compact $M$ as the traditional Hodge Laplacian $\triangle$ (Theorem 5.12).

The weighted Laplacians corresponding to $d \mu=e^{2 h} d x$ and $d \mu^{-}=e^{-2 h} d x$ satisfy a duality which is analogous to Poincaré duality on orientable, noncompact manifolds (Theorem 3.1).

If $\triangle_{\mu}$ has a spectral gap, then a strong Hodge decomposition of $L_{\mu}^{2} \Omega^{\bullet}$ can be found (Theorem 5.10).

The spectral properties of $\triangle_{\mu}$ on noncompact $M$ depend fundamentally on the growth or decay at infinity of the density of $d \mu$. One method for seeing this dependence is to note that for any $d \mu, \triangle_{\mu}$ acting in $L_{\mu}^{2} \Omega^{\bullet}$ is unitarily equivalent to $\triangle+V_{\mu}$ for a certain potential $V_{\mu}$, acting in unweighted $L^{2} \Omega^{\bullet}$ (Theorem 4.2).

Such a "Schrödinger operator" form of certain weighted Laplacians was considered by E. Witten [Wi] in his work on Morse theory on compact manifolds, which was further explored by J. Bismut [Bi]. K. Elworthy and S. Rosenberg [ER] used a weighted Laplacian to address the Hopf conjecture.

The Schrödinger operator form allows us to analyse the spectrum of $\triangle_{\mu}$ in some cases. Theorem 4.5 shows that if $V_{\mu}$ indeed behaves globally like a quadratic potential in the simplest sense that it goes to infinity at infinity on $M$, then $\triangle_{\mu}$ has compact resolvent, and thus a spectral gap.

If $d \mu$ is the heat kernel measure, or has similar behavior at infinity, then the potential $V_{\mu}$ appears to be comparable to the quadratic harmonic oscillator potential. Section 8 gives conditions on the heat kernel, in the form of conditions on $V_{\mu}$, which would be sufficient to prove that $\triangle_{\mu}$ has compact resolvent. Determining whether these conditions are satisfied in particular cases would seem to depend on improvements in the current state of "heat kernel estimates" technology.

If $M$ is a complete Riemannian manifold with a nice distance function $r(x)=$ $d(x, S)$, then it is natural to consider the measure $d \mu=\exp \left(-r^{2}\right) d x$, which shares the asymptotic behavior at large $r$ of the heat kernel measure. In Section 9 we show that with this choice of measure $\triangle_{\mu}$ has compact resolvent on two interesting classes of manifolds (Theorems 9.1 and 9.2).

Even more strikingly, a measure $d \mu$ with $\exp \left(-r^{2}\right)$ density along flat cylindrical ends allows us to show that every topologically tame, noncompact, orientable manifold $M$ has a metric $g$ and measure $d \mu$ such that a strong Hodge decomposition holds for $\triangle_{\mu}$ (Theorem 10.2). It is natural to conjecture that on such a manifold (topologically tame and with flat cylindrical ends) a strong Hodge decomposition also holds for $\triangle_{\mu}$ if $d \mu$ is the heat kernel measure.

This completes our list of evidence for Conjecture 1.1.

If $M$ is a complete manifold and $d \mu$ is a heat kernel measure, then $\triangle_{\mu}$ always exists and is unique up to precisely two choices, namely the basepoint $x_{0} \in M$ and time $t>0$. In this sense, the heat kernel weighted Hodge Laplacian $\triangle_{\mu}$ is a natural object on a complete manifold. Theorems $6.1,6.4$, and 7.1 are specific evidence that $\triangle_{\mu}$ deserves consideration. We are not certain, however, that $\triangle_{\mu}$ is superior to other $\triangle_{\mu^{\prime}}$ given by measures $d \mu^{\prime}$ with densities of comparable decay at infinity to that of $d \mu$.

The heat kernel measure $\rho_{t}\left(x_{0}, x\right) d x$ is of particular interest because infinitedimensional analogs of this measure seem likely to be natural objects for some interesting classes of infinite-dimensional manifolds. See [DriL] for the heat kernel measure on loop groups. See also the related work [AM], [K1], [K2], and [K3]. 
In fact, Theorem 7.1 for $\triangle_{\mu}$ on $M=R^{n}$ extends to an infinite-dimensional linear space in the following sense. If $B$ is an abstract Wiener space, so in particular $B$ possesses a Gaussian measure, and if $\triangle_{B}$ is the analog of $\triangle_{\mu}$, then $\triangle_{B}$ has a spectral gap, ker $\triangle_{B}^{0}=\{$ constants $\}$, and ker $\triangle_{B}^{p}=0$ for $p>0$. See Theorem 7.5, a result of I. Shigekawa [Sh]. The corresponding result for $d \mu^{-}$naturally fails to generalize in this manner.

Briefly, we now summarize what is known for $\triangle=d^{*} d+d d^{*}$, the traditional Hodge Laplacian, beyond the successful and well-known compact, boundary-less case. If $M$ is compact with boundary, then appropriate modifications lead to a successful theory. See [RaS] or [Cha1] for a summary. On certain complete noncompact $M$, for instance on hyperbolic manifolds ([Don]), rotationally-symmetric manifolds ([Dod] $)$, and large classes of noncompact manifolds with either negative ([DonX]) or positive ([EF2]) curvature, the spectral properties of $\triangle$ are understood to varying degrees. However, these known cases suggest that the spectral properties of $\triangle$ on complete, noncompact manifolds are far from those of compact $M$. The surveys $[\mathrm{A}]$ and $[\mathrm{L}]$ review many of these facts.

In fact, if $M$ is not compact, then the fundamental isomorphism of Hodge theory (False!)

$$
\mathcal{H}^{p} \cong H_{\text {de Rham }}^{p}(M), \quad M \text { not compact, }
$$

is just not true. In some cases, i.e. if $\triangle^{p}$ has a spectral gap, the right hand side can be replaced with an $L^{2}$-cohomology:

$$
\mathcal{H}^{p} \cong L^{2} H^{p} \quad \text { if } \triangle^{p} \text { has a spectral gap. }
$$

We define the cohomology vector space $L^{2} H^{p}$ in Section 5, and then we prove (1.1) — such an isomorphism is traditionally called a "strong Hodge decomposition." There is no a priori reason at all to believe $L^{2} H^{p} \cong H_{\text {de Rham }}^{p}(M)$, and in fact such an isomorphism always fails on noncompact $M$ in at least one of the cases $p=0$ or $p=n$.

Obviously, no square-integrable harmonic functions exist on Euclidean space $R^{n}$, even though $b_{0}=1$. Furthermore, (1.1) fails on $R^{n}$. The dimensions of ker $\triangle^{p}$ differ from the Betti numbers $b_{p}=\operatorname{dim} H_{p}(M)$ on hyperbolic space even though (1.1) holds ([Don]). Other "bad" examples are in generous supply-see [A], [Dod] and $[\mathrm{L}]$.

Indeed, $\triangle$ always possesses a symmetry

$$
\operatorname{ker} \triangle^{p} \cong \operatorname{ker} \triangle^{n-p}
$$

which is at odds with the topological reality for noncompact manifolds

$$
H_{\text {de Rham }}^{p}(M) \cong\left[H_{\text {de R,c }}^{n-p}(M)\right]^{*}
$$

(see Section 3). For instance, if $M^{n}$ is connected and noncompact, $H_{\text {de }}^{0}$ Rham $\left(M^{n}\right) \cong$ $R$ and $H_{\text {de Rham }}^{n}\left(M^{n}\right) \cong 0$, while ker $\triangle^{0} \cong \operatorname{ker} \triangle^{n}$.

The weighted Laplacians break the symmetry (1.2). Such symmetry breaking is required by (1.3), if Conjecture 1.1 is true. See Section 3 .

This research was motivated by a suggestion of L. Gross that the heat kernel measure was the appropriate finite-dimensional analog of the Gaussian measures used in the few infinite-dimensional cases of Hodge theory currently understood ([Gro]). Professor Gross directed the author to the results of $[\mathrm{Hs}]$ and $[\mathrm{DriH}]$, as well as suggesting the possibility of a construction like that given in Theorem 10.2. Thanks to M. Braverman and to an anonymous referee for helpful comments. 


\section{Definitions and Notation}

Let $M$ be a connected, oriented, and complete Riemannian manifold of dimension $n$, such that $\partial M=\emptyset$. The connectedness and orientability of $M$ are largely matters of convenience, but the completeness of $M$ will be essential. Our interest is in the case of noncompact $M$, but our results will apply to the compact case.

Denote the space of smooth differential $p$-forms by $\Omega^{p}$ and those with compact support by $\Omega_{c}^{p}$. Let $d^{p}$ be the usual exterior derivative on $\Omega^{p}$. Write $\Omega^{\bullet}$ for $\bigoplus_{q=0}^{n} \Omega^{q}$, the space of forms, and $\Omega_{c}^{\bullet}$ for the space of compactly supported forms.

Let $h$ be a $C^{\infty}$ real function on $M$, and define

$$
d \mu(x)=e^{2 h(x)} d x,
$$

a measure on $M$ absolutely continuous with respect to Riemann-Lebesgue measure $d x$. This will be the measure used in defining the weighted Laplacian, which we will denote by $\triangle_{\mu}$ and define below. We will work with both the function $h=\frac{1}{2} \ln \frac{d \mu}{d x}$ and the measure $d \mu$.

As a particular case of such a measure, we will be interested in a heat kernel $\rho_{t}\left(x_{0}, \cdot\right)$, for a fixed point $x_{0} \in M$ and fixed time $t>0$, and in the associated finite measure

$$
d \mu(x)=\rho_{t}\left(x_{0}, x\right) d x .
$$

We define $\rho$ as the normalized fundamental solution to $\frac{\partial \rho}{\partial t}=-\Delta \rho$ - see [Da1] for the existence proof. We take the ordinary Laplace-Beltrami operator $\triangle$ as nonnegative, so $\triangle=\delta d$ in the notation which follows.

We now begin with the serious business of constructing a Hilbert space theory of the de Rham complex, i.e. a Hilbert space version of

$$
\cdots \stackrel{d^{p-2}}{\longrightarrow} \Omega^{p-1} \stackrel{d^{p-1}}{\longrightarrow} \Omega^{p} \stackrel{d^{p}}{\longrightarrow} \Omega^{p+1} \quad \stackrel{d^{p+1}}{\longrightarrow} \cdots
$$

which, as it stands, is a sequence of maps on noncomplete spaces of smooth forms with no natural inner product (in the noncompact case). We will do a natural thing - we will define an inner product and complete some nice space in this inner product to get a Hilbert space. The resulting complex is naturally called a Hilbert complex.

First, there is a standard pointwise inner product on forms on a Riemannian manifold $M$ :

$$
(\phi, \psi)_{x}=\star(\phi(x) \wedge \star \psi(x)),
$$

if $\phi, \psi$ are $p$-forms. If $\phi, \psi$ differ in degree, then we define $(\phi, \psi)_{x}=0$. Note the use of the Hodge star operator $\star: \Omega^{p} \rightarrow \Omega^{n-p}$, the definition of which involves both an orientation and a metric on $M$. (The text by F. Warner [Wa] is a reference for these and many other standard facts about forms.)

For every $\phi, \psi \in \Omega_{c}^{p}$, let

$$
\langle\phi, \psi\rangle_{\mu}=\int_{M}(\phi, \psi)_{x} d \mu(x)
$$

If $d \mu=d x$, then this is just the standard inner product on forms, frequently written

$$
\langle\phi, \psi\rangle=\int_{M} \phi \wedge \star \psi .
$$


Define $L_{\mu}^{2} \Omega^{p}$ as the completion of $\Omega_{c}^{p}$ in the norm $\|\cdot\|_{\mu}=\sqrt{\langle\cdot, \cdot\rangle_{\mu}}$. In fact, $L_{\mu}^{2} \Omega^{p}$ is a space of equivalence classes (up to measure zero) of measurable sections of the exterior $p$ bundle over $M$. The inner product extends orthogonally to all of

$$
L_{\mu}^{2} \Omega^{\bullet}=\bigoplus_{p=0}^{n} L_{\mu}^{2} \Omega^{p} .
$$

Now, $d^{p}: \Omega_{c}^{p} \rightarrow \Omega_{c}^{p+1}$ is a densely-defined operator in $L_{\mu}^{2} \Omega^{p}$. It has a well-known formal adjoint $\delta$ with respect to the inner product for $d \mu=d x$. Given $\phi \in \Omega_{c}^{p}$ and $\psi \in \Omega_{c}^{p+1}$,

$$
\begin{aligned}
\left\langle d^{p} \phi, \psi\right\rangle_{\mu} & =\int_{M} d^{p} \phi \wedge \star \psi e^{2 h} d x=\int_{M} \phi \wedge \star \delta^{p+1}\left(\psi e^{2 h}\right) d x \\
& =\left\langle\phi,\left(e^{-2 h} \delta^{p+1} e^{2 h}\right) \psi\right\rangle_{\mu},
\end{aligned}
$$

so $e^{-2 h} \delta e^{2 h}$ is the (formal) adjoint of $d$ in $L_{\mu}^{2} \Omega^{\bullet}$. (Concretely, $\delta$ has the formula $\delta^{p+1}=(-1)^{n(p+2)+1} \star d^{n-p-1} \star$ on $\Omega_{c}^{p+1}$ - see [Wa], p. 220.)

Consider $d$ and $\delta$ as acting on $\Omega_{c}^{\bullet}$. Let

$$
\delta_{\mu}=e^{-2 h} \delta e^{2 h}=\delta-2 i_{\nabla h} .
$$

Note $\delta(f \omega)=-i_{\nabla f} \omega+f \delta \omega$, where $i_{X}$ is interior multiplication of forms by the vector field $X$. Also $\left(\delta_{\mu}\right)^{2}=0$ since $d^{2}=0$.

Let

$$
D_{\mu}=d+\delta_{\mu}
$$

with domain $\Omega_{c}^{\bullet}$. Note that $D_{\mu}$ has no degree, but that it sends forms of odd order to forms of even order, and vice versa. Its square

$$
D_{\mu}^{2}=d^{2}+d \delta_{\mu}+\delta_{\mu} d+\delta_{\mu}^{2}=d \delta_{\mu}+\delta_{\mu} d
$$

is of degree zero. Furthermore,

$$
D_{\mu}^{2}=d \delta_{\mu}+\delta_{\mu} d=\triangle-2 L_{\nabla h},
$$

where $\triangle=d \delta+\delta d$ and $L_{X}=d i_{X}+i_{X} d$ is Lie differentiation in the direction (vector field) $X$.

Define

$$
\triangle_{\mu}=D_{\mu}^{2}=d \delta_{\mu}+\delta_{\mu} d=\triangle-2 L_{\nabla h}
$$

a densely-defined (i.e. on $\Omega_{c}^{\bullet}$ ) degree zero operator in $L_{\mu}^{2} \Omega^{\bullet}$. Note that on functions $f, L_{X} f=X f$, so

$$
\triangle_{\mu}^{0}=\triangle^{0}-2 \nabla h .
$$

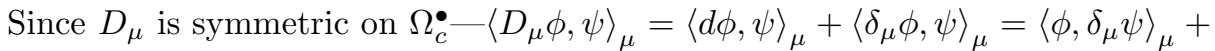
$\langle\phi, d \psi\rangle_{\mu}=\left\langle\phi, D_{\mu} \psi\right\rangle_{\mu}$-it is closeable. Similarly $\triangle_{\mu}=D_{\mu}^{2}$ is symmetric on $\Omega_{c}^{\bullet}$ and thus closeable. With our conventions,

$$
\left\langle\triangle_{\mu} \phi, \phi\right\rangle_{\mu}=\|d \phi\|_{\mu}^{2}+\left\|\delta_{\mu} \phi\right\|_{\mu}^{2}=\left\langle D_{\mu} \phi, D_{\mu} \phi\right\rangle_{\mu} \geq 0
$$

for $\phi \in \Omega_{c}^{\bullet}$.

It is clear that $\overline{D_{\mu}}=\bar{d}+\overline{\delta_{\mu}}$ since

$$
\operatorname{im} d \perp \operatorname{im} \delta_{\mu} \quad \text { in } \quad L_{\mu}^{2} \Omega^{p} .
$$


We define the two closed operators: $d=\bar{d}$ and $d_{\mu}^{*}=\overline{\delta_{\mu}}$. Of course, $(d)^{*}=d_{\mu}^{*}$ with respect to the inner product $\langle\cdot, \cdot\rangle_{\mu}$. By our conventions, $\overline{\delta_{\mu}^{p+1}}=\left(d^{p}\right)^{*}=\left(d_{\mu}^{*}\right)^{p+1}$.

By a slight abuse of notation, we define $\triangle_{\mu}$ to be the closure of $\triangle_{\mu}$ on $\Omega_{c}^{\bullet}$.

\subsection{Definition.}

$$
\triangle_{\mu} \text { is the closure of } d \delta_{\mu}+\delta_{\mu} d \text { on } \Omega_{c}^{\bullet} \text {. }
$$

The nonobvious identity $\triangle_{\mu}=d d_{\mu}^{*}+d_{\mu}^{*} d$ remains to be proved. We will do so in Lemma 5.2.

Moreover, in Theorem 4.3 we will prove that $\triangle_{\mu}$ is self-adjoint.

\section{Poincaré Duality in the Weighted Case}

Let $h$ be a smooth function on $M^{n}$. Let $L_{\mu}^{2} \Omega^{\bullet}$ be the Hilbert space of forms corresponding to the measure $d \mu=e^{+2 h} d x$ as before, and introduce $L_{\mu^{-}}^{2} \Omega^{\bullet}$ corresponding to the measure $d \mu^{-}=e^{-2 h} d x$. Define $\triangle_{\mu}$ and $\triangle_{\mu^{-}}$by the procedure of Section 2, using the respective measures. Recall that the Hodge $\star$ operator is defined on orientable $(M, g)$.

3.1 Theorem [ER].

$$
\star_{\mu}: \omega \mapsto e^{2 h} \star \omega
$$

is a unitary map from $L_{\mu^{2}}^{2} \Omega^{p}$ to $L_{\mu^{-}}^{2} \Omega^{n-p}$, and

$$
\star_{\mu} \triangle_{\mu}^{p}=\triangle_{\mu^{-}}^{n-p} \star_{\mu}
$$

on $\Omega_{c}^{p}$.

\subsection{Corollary.}

$$
\operatorname{ker} \triangle_{\mu}^{p} \cong \operatorname{ker} \triangle_{\mu^{-}}^{n-p} .
$$

Theorem 3.1 is a weighted version of the relation $\star \Delta=\Delta \star$, a symmetry which yields a quick proof, via the theorems of Hodge and de Rham, of Poincaré duality on a compact orientable manifold. See [Wa], pp. 226-227. The symmetry $\star \Delta=\triangle \star$ and its corollary ker $\triangle^{p} \cong \operatorname{ker} \triangle^{n-p}$ apply on noncompact $M$. Theorem 3.3 below shows the desirability of breaking this symmetry in the noncompact case.

3.3 Theorem (Poincaré duality on noncompact $M$ ). If $M^{n}$ is orientable, then

$$
H_{\text {de Rham }}^{p}(M) \cong\left[H_{\text {de R,c }}^{n-p}(M)\right]^{*} .
$$

Theorems 6.1 and 6.2 imply that if $d \mu$ is the heat kernel measure on a noncompact manifold with $\operatorname{Ric}_{M} \geq 0$, then $\operatorname{ker} \triangle_{\mu}^{0} \neq \operatorname{ker} \triangle_{\mu}^{n}$. The same theorems also show that $\triangle_{\mu}$ is a different operator from $\triangle^{\prime}$, where $\triangle^{\prime}$ is the ordinary Hodge Laplacian for $\left(M, g^{\prime}=e^{(4 h) / n} g\right)$-the conformal change of metric which replaces $d x$ by $d x^{\prime}=$ $e^{2 h} d x$.

Poincaré duality follows from showing that the (bilinear) pairing $\omega, \nu \mapsto \int_{M} \omega \wedge \nu$ for $\omega \in \Omega^{p}$ and $\nu \in \Omega_{c}^{n-p}$ descends to a nondegenerate pairing on cohomology. In this spirit, we offer 
3.4 Theorem. The continuous, bilinear pairing

$$
\begin{aligned}
L_{\mu}^{2} \Omega^{p} \times L_{\mu^{-}}^{2} \Omega^{n-p} & \rightarrow R \\
\omega, \nu & \mapsto \int_{M} \omega \wedge \nu
\end{aligned}
$$

descends to a nondegenerate pairing on $L_{\mu}^{2}$-cohomology. (See Section 5 for the definition of the $L_{\mu}^{2}$-cohomology vector spaces $L_{\mu}^{2} H^{p}$.) That is, we have a nondegenerate continuous bilinear map

$$
L_{\mu}^{2} H^{p} \times L_{\mu^{-}}^{2} H^{n-p} \quad \rightarrow \quad R .
$$

Proof. In fact,

$$
\begin{aligned}
\left|\int_{M} \omega \wedge \nu\right| & =\left|\int_{M}\left(e^{h} \omega\right) \wedge \star\left(e^{-h}(-1)^{p(n-p)} \star \nu\right)\right| \\
& \leq\left\|e^{h} \omega\right\|_{d x}\left\|e^{-h} \star \nu\right\|_{d x}=\|\omega\|_{\mu}\|\nu\|_{\mu^{-}}
\end{aligned}
$$

so the pairing is continuous on the $L_{\mu}^{2}$-spaces of forms.

Descent to cohomology requires $\int_{M} d \omega \wedge \nu=0$ for $\omega \in \mathcal{D}_{d \mid L_{\mu}^{2} \Omega^{p-1}}$ and $\nu \in$ $\left.\operatorname{ker} d\right|_{L_{\mu^{2}} \Omega^{n-p}}$. This needs proof in our $L_{\mu}^{2}$ setting, as follows. There are sequences $\omega_{j} \in \Omega_{c}^{p-1}$ converging to $\omega$ and $\nu_{k} \in \Omega_{c}^{n-p}$ converging to $\nu$, in the graph norm of $d$, on $L_{\mu}^{2} \Omega^{p-1}$ and $L_{\mu^{-}} \Omega^{n-p}$, respectively. Furthermore, $d \nu_{k} \rightarrow 0$, of course. Now,

$$
d \omega \wedge \nu=d \omega_{j} \wedge \nu_{k}+\left(d \omega-d \omega_{j}\right) \wedge \nu_{k}+d \omega_{j} \wedge\left(\nu-\nu_{k}\right)+\left(d \omega-d \omega_{j}\right) \wedge\left(\nu-\nu_{k}\right)
$$

and

$$
\int_{M} d \omega_{j} \wedge \nu_{k}=\int_{M} d\left(\omega_{j} \wedge \nu_{k}\right)+(-1)^{p} \int_{M} \omega_{j} \wedge d \nu_{k} \rightarrow 0
$$

Apply (3.1) to show $\int_{M} d \omega \wedge \nu=0$. Similarly, it follows that $\int_{M} \omega \wedge d \nu=0$ if $\omega$ is closed.

Finally, to show the pairing on $L_{\mu}^{2}$-cohomology is nondegenerate, take $\omega \in$ $\left.\operatorname{ker} d\right|_{L_{\mu}^{2} \Omega^{p}}$ which is not exact (i.e. not in the image of $d$ on $L_{\mu}^{2} \Omega^{p-1}$ ). Clearly, if $\nu=e^{2 h} \star \omega$, then $\int_{M} \nu \wedge \omega=\|\omega\|_{\mu}^{2}>0$. But then $\nu$ is not exact, as shown above.

We have shown exactly that

$$
\nu=e^{2 h} \star \omega \text { is the Poincaré dual of } \omega
$$

in our weighted theory.

\section{Weighted Laplacian as Schrödinger Operator}

The fundamental results of this section are that the weighted Laplacian $\triangle_{\mu}$ acting in $L_{\mu}^{2} \Omega^{\bullet}$ is unitarily equivalent to $\triangle+V_{\mu}$ acting in $L^{2} \Omega^{\bullet}$ for a potential $V_{\mu}$ (Theorem 4.2) and that $\triangle_{\mu}$ has compact resolvent if $V_{\mu}$ goes to infinity at infinity (Theorem 4.5).

The unitary equivalence between $\triangle_{\mu}$ and $\triangle+V_{\mu}$ is naturally related to the "ground state transform" in quantum physics. Consider a Schrödinger operator

$$
H=-\frac{d^{2}}{d x^{2}}+V(x) \quad \text { in } \quad L^{2}\left(R^{1}\right)
$$


possessing both a simple lowest eigenvalue $\lambda_{0}=0$ and a smooth positive ground state $\varphi_{0}$. The ground state transform is the unitary map $U: f \mapsto \varphi_{0} f$ which maps $L_{\varphi_{0}^{2} d x}^{2}\left(R^{1}\right) \rightarrow L^{2}\left(R^{1}\right)$, where $\varphi_{0}^{2} d x$ is the measure for the weighted $L^{2}$-space $L_{\varphi_{0}^{2} d x}^{2}\left(R^{1}\right)$ with inner product

$$
f, g \mapsto \int_{R^{1}} f g \varphi_{0}^{2} d x
$$

The operator $H$ is then unitarily equivalent to $N=U^{-1} H U$ acting in $L_{\varphi_{0}^{2} d x}^{2}\left(R^{1}\right)$; the reader may verify that

$$
N f=\varphi_{0}^{-1} H\left(\varphi_{0} f\right)=\left(-\frac{d}{d x}-\left(2 \ln \varphi_{0}\right)^{\prime}\right) \frac{d}{d x} f .
$$

Significantly, $N$ is a Dirichlet form operator, that is

$$
\int_{R^{1}} f(N f) \varphi_{0}^{2} d x=\int_{R^{1}}\left(f^{\prime}\right)^{2} \varphi_{0}^{2} d x
$$

In this section we will think of the weighted Laplacian $\triangle_{\mu}$ as $N$, and we will find the corresponding $H$. If one starts (as we have) with a measure of the form $e^{2 h} d x$, then (thinking " $\varphi_{0}=e^{h}$ "), we may "undo the ground state transform". This is done in Lemma 4.1 and Theorem 4.2 below. Note that we are ultimately interested in the measure $d \mu=\rho_{t}\left(x_{0}, x\right) d x$, for which the "ground state" is $\varphi_{0}(x)=\sqrt{\rho_{t}\left(x_{0}, x\right)}$.

4.1 Lemma. Let $d \mu=e^{2 h} d x$ on $M$. The map

$$
\begin{aligned}
U: L_{\mu}^{2} \Omega^{\bullet} & \rightarrow L^{2} \Omega^{\bullet}, \\
\omega & \mapsto e^{h} \omega
\end{aligned}
$$

is unitary and degree preserving.

Proof. Clear.

For $f \in C^{\infty}(M)$, following [EF2] we define the Hessian $\mathcal{H}_{f}$ acting on forms by

$$
\left(\mathcal{H}_{f} \omega\right)(x)=\sum_{i, j=1}^{n} H f\left(X_{i}, X_{j}\right) \phi^{j} \wedge i_{X_{i}} \omega,
$$

where $\left\{X_{i}\right\}_{i=1}^{n}$ is an orthonormal basis of $T_{x} M,\left\{\phi^{i}\right\}_{i=1}^{n}$ is its dual basis, and where

$$
H f(X, Y)=X Y f-\left(\nabla_{X} Y\right) f
$$

is the classical Hessian of $f$. Note $\left.\mathcal{H}_{f}\right|_{x}$ is a symmetric operator on $\wedge^{\bullet} T_{x}^{*} M$ with respect to $(\cdot, \cdot)_{x}$.

4.2 Theorem ([Wi] and [Bi] $)$. Let $D_{\mu}^{U}=e^{h}\left(d+d_{\mu}^{*}\right) e^{-h}=e^{h} d e^{-h}+e^{-h} \delta e^{h}$ and let $\triangle_{\mu}^{U}=\left(D_{\mu}^{U}\right)^{2}$, as operators in $L^{2} \Omega^{\bullet}$. $D_{\mu}^{U}$ and $\triangle_{\mu}^{U}$ are unitarily equivalent to $D_{\mu}$ and $\triangle_{\mu}$ acting in $L_{\mu}^{2} \Omega^{\bullet}$. If $\xi \in \Omega_{c}^{\bullet}$, then

$$
\begin{aligned}
& D_{\mu}^{U} \xi=(d+\delta) \xi-d h \wedge \xi-i_{\nabla h} \xi \\
& \triangle_{\mu}^{U} \xi=\triangle \xi+|d h|^{2} \xi-\left(\triangle^{0} h\right) \xi-2 \mathcal{H}_{h} \xi
\end{aligned}
$$

Proof. A calculation. We use the formulae

$$
\begin{gathered}
d f \wedge\left(i_{\nabla f} \omega\right)+i_{\nabla f}(d f \wedge \omega)=|d f|^{2} \omega, \\
\delta(d f \wedge \omega)=\left(\triangle^{0} f\right) \omega+\mathcal{H}_{f} \omega-\nabla_{\nabla f} \omega-d f \wedge \delta \omega,
\end{gathered}
$$


and

$$
L_{\nabla f}=\nabla_{\nabla f}+\mathcal{H}_{f}
$$

Here $\nabla$ is the Levi-Civita covariant differentiation acting on tensors (forms) and (as before) $L_{X}=i_{X} d+d i_{X}$ is the Lie derivative. The paper by J. Escobar and A. Freire [EF2] is a most useful reference for such formulae.

Then

$$
\begin{aligned}
D_{\mu}^{U} \xi & =e^{h} d\left(e^{-h} \xi\right)+e^{-h} \delta\left(e^{h} \xi\right) \\
& =e^{h}\left(d\left(e^{-h}\right) \wedge \xi+e^{-h} d \xi\right)+e^{-h}\left(-i_{\nabla\left(e^{h}\right)} \xi+e^{h} \delta \xi\right) \\
& =(d+\delta) \xi-d h \wedge \xi-i_{\nabla h} \xi,
\end{aligned}
$$

and

$$
\begin{aligned}
\triangle_{\mu}^{U} \xi & =-e^{h} d\left(e^{-h} i_{\nabla h} \xi\right)+e^{h} d\left(e^{-h} \delta \xi\right)-e^{-h} \delta\left(e^{h} d h \wedge \xi\right)+e^{-h} \delta\left(e^{h} d \xi\right) \\
& =\triangle \xi+|d h|^{2} \xi-\left(\triangle^{0} h\right) \xi-\mathcal{H}_{h} \xi-L_{\nabla h} \xi+\nabla_{\nabla h} \xi,
\end{aligned}
$$

as desired.

We see that $\triangle_{\mu}$ is unitarily equivalent to $\triangle_{\mu}^{U}=\triangle+V_{\mu}$, for $V_{\mu}=|d h|^{2}-\left(\triangle^{0} h\right)-$ $2 \mathcal{H}_{h}$. We will show that $\triangle_{\mu}^{U}$ is essentially self-adjoint in $L^{2} \Omega^{\bullet}$.

It is well known that if $M$ is complete, then $\triangle$ with dense domain $\Omega_{c}^{\bullet}$ is essentially self-adjoint in $L^{2} \Omega^{\bullet}$. The original proof can be found in [Ga].

However, P. Chernoff's [Che] proof of this same fact extends to prove that $\triangle_{\mu}^{U}$ is essentially self-adjoint. Specifically, Chernoff's method shows that both $D=d+\delta$ and $D_{\mu}^{U}=d+\delta-d h \wedge-i_{\nabla h}$ are essentially self-adjoint on $\Omega_{c}^{\bullet}$ in $L^{2} \Omega^{\bullet}$. The identical argument applies to both cases because the method depends only on the symbols of the operators. That is, $D_{\mu}^{U}=D+$ (zero-order terms), and smooth, symmetric zeroorder terms are irrelevant to the essential self-adjointness of a first-order differential operator on a complete manifold. Chernoff then shows that every positive, integer power of either $D$ or $D_{\mu}^{U}$ is essentially self-adjoint.

4.3 Theorem ([Che], Theorem 2.2). Assume $M$ is complete. Then $D_{\mu}^{U}$ and $\triangle_{\mu}^{U}$ are essentially self-adjoint on $\Omega_{c}^{\bullet}$, as unbounded operators in $L^{2} \Omega^{\bullet}$. It follows that $D_{\mu}$ and $\triangle_{\mu}$ are essentially self-adjoint on $\Omega_{c}^{\bullet}$, in $L_{\mu}^{2} \Omega^{\bullet}$.

As the square of a self-adjoint operator, $\triangle_{\mu}^{U}$ is nonnegative.

Now consider the form of $\triangle_{\mu}^{U}$ when $\rho=e^{2 h}$ is the heat kernel and $M=R^{n}$. In particular (taking $x_{0}=0$ and any $t>0$ )

$$
\rho_{t}(x, 0)=(4 \pi t)^{n / 2} e^{-x^{2} / 4 t},
$$

so $|d h|^{2}=\frac{x^{2}}{16 t^{2}}, \triangle^{0} h=\frac{n}{4 t}$, and

$$
\triangle_{\mu}^{U}=\triangle+\frac{x^{2}}{16 t^{2}}-\frac{n}{4 t}-2 \mathcal{H}_{h}
$$

On functions ( $p=0$ forms), $\mathcal{H}_{f}=0$ for any $f$ so

$$
\triangle_{\mu}^{U, 0}=-\sum_{i=1}^{n} \frac{\partial^{2}}{\partial x_{i}^{2}}+\frac{x^{2}}{16 t^{2}}-\frac{n}{4 t} .
$$

Thus $\triangle_{\mu}^{U, 0}$ is a Schrödinger operator, in the usual sense, with quadratic potential. And $\triangle_{\mu}^{U, 0}$ has lowest eigenvalue $\lambda=0$ because $\triangle_{\mu}^{U, 0}$ on $L^{2}\left(R^{n}\right)$ is unitarily 
equivalent to $\triangle_{\mu}^{0}=\delta_{\mu} d$ on $L_{\mu}^{2}\left(R^{n}\right)$, which has constant functions in its kernel since $d \mu$ is a finite measure.

Compare (4.3) to the Hamiltonian of a quantum harmonic oscillator. In one dimension,

$$
H=-\frac{\hbar^{2}}{2} \frac{\partial^{2}}{\partial x^{2}}+\frac{x^{2}}{2} \quad \text { on } \quad L^{2}\left(R^{1}\right) .
$$

One traditionally removes the ground state energy and rescales to get a number operator $N$ with spectrum $\{0,1,2, \ldots\}$. In fact,

$$
\frac{2}{\hbar} N=\frac{2}{\hbar^{2}} H-\frac{1}{\hbar}=-\frac{\partial^{2}}{\partial x^{2}}+\frac{x^{2}}{\hbar^{2}}-\frac{1}{\hbar}
$$

is exactly

$$
\triangle_{\mu}^{U, 0}=-\frac{\partial^{2}}{\partial x^{2}}+\frac{x^{2}}{(4 t)^{2}}-\frac{1}{(4 t)}
$$

if we identify $\hbar=4 t$. (We take an agnostic view of this identification.)

Next, we note that $\triangle_{\mu}^{U}$ is the weighted Laplacian featured in E. Witten's treatment of Morse theory on compact $M$ ([Wi]). Witten writes $V_{\mu}$ in a different form, which we outline here. We will use both forms according to convenience.

Let $x \in M$. Let $\left\{X_{i}\right\}_{i=1}^{n}$ be an orthonormal basis of $T_{x} M$ and let $\left\{\phi^{i}\right\}_{i=1}^{n}$ be its dual basis. Let $\xi \in \wedge^{\bullet} T_{x}^{*} M$. Note $\triangle^{0} f=-\operatorname{tr} H f$ and $\phi^{i} \wedge\left(i_{X_{j}} \xi\right)+i_{X_{j}}\left(\phi^{i} \wedge \xi\right)=$ $\delta^{i j} \xi$, so

$$
\begin{aligned}
-\left(\triangle^{0} h\right) \xi-2 \mathcal{H}_{h} \xi & =\sum_{i, j} H h\left(X_{i}, X_{j}\right)\left(\delta^{i j} \xi-2 \phi^{i} \wedge i_{X_{j}} \xi\right) \\
& =\sum_{i, j} H h\left(X_{i}, X_{j}\right)\left(\left[i_{X_{j}}, \phi^{i} \wedge\right]\right) \xi .
\end{aligned}
$$

Rewriting $V_{\mu}$ gives:

$$
V_{\mu}=|d h|^{2}+\sum_{i, j} H h\left(X_{i}, X_{j}\right)\left[a^{j}, a^{i *}\right]
$$

where $a^{i *}=\phi^{i} \wedge, a^{j}=i_{X_{j}}$ are "fermion creation and annihilation operators." Of course, $V_{\mu}$ acts in a well-defined manner on (global) sections of $\wedge^{\bullet} T^{*} M$, i.e. on differential forms. Note also that

$$
\left[a^{i}, a^{i *}\right] \phi^{k_{1}} \wedge \cdots \wedge \phi^{k_{p}}= \begin{cases}-\phi^{k_{1}} \wedge \cdots \wedge \phi^{k_{p}} & \text { if } i \in\left\{k_{1}, \ldots, k_{p}\right\} \\ +\phi^{k_{1}} \wedge \cdots \wedge \phi^{k_{p}} & \text { if } i \notin\left\{k_{1}, \ldots, k_{p}\right\}\end{cases}
$$

in keeping with the "creation" and "annihilation" operator meaning. This is Witten's language - in fact, the form of $V_{\mu}$ is at the heart of his rederivation of the Morse inequalities on a compact manifold. See [Wi], pp. 665-666, and [CFKS], pp. 233-234.

To view $\triangle_{\mu}^{U}$ as a Schrödinger operator, we must generalize our concept of "potential" slightly, from the traditional situation in which a potential is a scalar multiplication operator. We include the term involving the Hessian of $h$ in our meaning of potential- $\left(\mathcal{H}_{h}\right)_{x}$ acts as an operator on the finite-dimensional space $\wedge^{\bullet} T_{x}^{*} M$. That is, $\mathcal{H}_{h}$ and $V_{\mu}$ are smooth, symmetric sections of $\operatorname{End}\left(\wedge^{\bullet} T^{*} M\right)$.

Now, in the case of the quantum harmonic oscillator with Hamiltonian $H=$ $-\frac{1}{2}\left(\frac{\partial}{\partial x}\right)^{2}+\frac{1}{2} x^{2}$, the potential $\frac{1}{2} x^{2}$ goes to infinity at infinity, and this property 
implies that $H$ has purely discrete spectrum consisting of a sequence of eigenvalues tending to infinity. That is, $H$ has compact resolvent. That

$$
V(x) \nearrow+\infty \text { at } \infty \text { implies } \triangle+V \text { has compact resolvent }
$$

also holds in our case, as we explain in Theorem 4.5.

Recall our intended context for this result. Closed balls $B_{R}(y)$ are compact-this is the Hopf-Rinow theorem (see [Cha2], p. 26). The "Schrödinger operator" $\triangle_{\mu}^{U}$ is essentially self-adjoint in $L^{2} \Omega^{\bullet}$ with core $\Omega_{c}^{\bullet}$. Since $\triangle_{\mu}^{U}=\left(D_{\mu}^{U}\right)^{2}$, it is nonnegative.

4.4 Lemma. Let $A$ be a nonnegative, self-adjoint (unbounded) operator in a Hilbert space. Suppose $\mathcal{C} \subset \mathcal{D}_{A}$ is a domain on which $A$ is essentially self-adjoint. Then $(1+A)^{-1}$ is compact iff

$$
\begin{aligned}
& \text { for every sequence }\left\{\psi_{n}\right\} \subset \mathcal{C} \text { such that }\left\|\psi_{n}\right\| \leq c \text { and }\left\|A \psi_{n}\right\| \leq c \text {, } \\
& \left\{\psi_{n}\right\} \text { has a Cauchy subsequence. }
\end{aligned}
$$

Proof. Let $\left\{\psi_{n}\right\}$ be a sequence as in (4.5). Note $\left\|(1+A) \psi_{n}\right\| \leq 2 c$. If $(1+A)^{-1}$ is compact, then $\left\{\psi_{n}=(1+A)^{-1}(1+A) \psi_{n}\right\}$ has a Cauchy subsequence.

Suppose (4.5) holds. Let $\left\{\varphi_{n}\right\}$ be bounded. Let $\xi_{n}=(1+A)^{-1} \varphi_{n} \in \mathcal{D}_{A}$. Choose $\left\{\psi_{n}\right\} \subset \mathcal{C}$ such that $\left\|\psi_{n}-\xi_{n}\right\|+\left\|A \psi_{n}-A \xi_{n}\right\| \leq \frac{1}{n}$. Note $\left\|\psi_{n}\right\| \leq \frac{1}{n}+\left\|(1+A)^{-1} \varphi_{n}\right\|$ is bounded. By the spectral theorem, $\left\|A \psi_{n}\right\| \leq \frac{1}{n}+\left\|\frac{A}{1+A} \varphi_{n}\right\|$ is bounded. Thus $\left\{\psi_{n}\right\}$ has a Cauchy subsequence, and so $\left\{\xi_{n}\right\}$ has one also.

4.5 Theorem. Let $V$ be a continuous, symmetric section of $\operatorname{End}\left(\wedge^{\bullet} T_{x}^{*} M\right)$. Assume

$$
H=\triangle+V
$$

is nonnegative and essentially self-adjoint on $\Omega_{c}^{\bullet}$. Assume that

$$
\nu(x) \equiv\left(\text { smallest eigenvalue of } V(x) \text { on } \wedge^{\bullet} T_{x}^{*} M\right) \nearrow+\infty \text { at } \infty,
$$

in the sense that there exists $y \in M$ such that for each $N>0$, there exists $R>0$ such that $\nu(x) \geq N$ if $x \notin B_{R}(y)$. Then

\section{$H$ has compact resolvent.}

Proof. Clearly $V$ is bounded below-assume that $\nu(x) \geq-1$.

Suppose $\left\{\psi_{n}\right\} \subset \Omega_{c}^{\bullet}$ satisfies $\left\|\psi_{n}\right\| \leq c$ and $\left\|H \psi_{n}\right\| \leq c$. Let $\epsilon>0$. We will find a subsequence $\left\{\psi_{n_{k}}\right\}$ of $\left\{\psi_{n}\right\}$ such that $\left\|\psi_{n_{k}}-\psi_{n_{l}}\right\|<\epsilon$ for sufficiently large $k, l$. By taking a sequence of $\epsilon$ tending to zero, and applying the usual diagonalization argument to the iterated subsequences, we will find a Cauchy subsequence of $\left\{\psi_{n}\right\}$. By Lemma 4.4 this proves $(1+A)^{-1}$ is compact.

Note

$$
\begin{aligned}
2 c^{2} & \geq\left\langle(1+H) \psi_{n}, \psi_{n}\right\rangle=\left\langle\Delta \psi_{n}, \psi_{n}\right\rangle+\int_{M}\left((1+V(x)) \psi_{n}, \psi_{n}\right)_{x} d x \\
& \geq \int_{M}\left((1+\nu(x)) \psi_{n}, \psi_{n}\right)_{x} d x \geq \int_{M \backslash B_{R}}(1+\nu(x))\left|\psi_{n}\right|_{x}^{2} d x .
\end{aligned}
$$

Choose $R>0$ large enough so that $1+\nu(x) \geq \frac{32}{\epsilon^{2}} c^{2}$ for all $x \in M \backslash B_{R}$. Then

$$
\int_{M \backslash B_{R}}\left|\psi_{n}\right|_{x}^{2} d x \leq \frac{\epsilon^{2}}{16}
$$

The following local fact is used to show $\triangle$ has compact resolvent on compact $M$ : if $\left\{\alpha_{n}\right\} \subset \Omega_{c}^{\bullet}$ satisfies $\left\|\alpha_{n}\right\| \leq c$ and $\left\|\triangle \alpha_{n}\right\| \leq c$, and if $m \in M$, then there 
is some neighborhood $U$ of $m$ such that for any $\varphi \in C_{c}^{\infty}(U),\left\{\varphi \alpha_{n}\right\}$ has a Cauchy subsequence. (Note that this is explicitly stated in [Wa], Chapter 6 , in proving Theorem 6.6.)

So let $\left(U_{i}, \varphi_{i}\right)_{i=1}^{I}$ be a partition of unity associated to a finite cover of $B_{R}$ by such neighborhoods. In particular, $\bar{\varphi}=\sum_{i=1}^{I} \varphi_{i}=1$ on $B_{R}$ and

$$
B_{R} \subset \operatorname{supp} \bar{\varphi} \subset \bar{U}=\bigcup_{i=1}^{I} U_{i} \subset M .
$$

Choose a subsequence of $\psi_{n},\left\{\psi_{n_{k}}\right\}$, for which each $\left\{\varphi_{i} \psi_{n_{k}}\right\}$ is Cauchy. Then $\left\{\bar{\varphi} \psi_{n_{k}}\right\}$ is Cauchy, and $\operatorname{supp} \bar{\varphi} \psi_{n_{k}} \subset \bar{U}$.

On the other hand,

$$
\left\|(1-\bar{\varphi}) \psi_{n_{k}}\right\|^{2}=\int_{M \backslash B_{R}}(1-\bar{\varphi})^{2}\left|\psi_{n_{k}}\right|_{x}^{2} d x \leq \int_{M \backslash B_{R}}\left|\psi_{n_{k}}\right|_{x}^{2} d x,
$$

so by (4.6),

$$
\left\|(1-\bar{\varphi}) \psi_{n_{k}}\right\|^{2} \leq \frac{\epsilon^{2}}{16}
$$

Choose $k, l$ large enough so that $\left\|\bar{\varphi} \psi_{n_{k}}-\bar{\varphi} \psi_{n_{l}}\right\| \leq \frac{\epsilon}{2}$. Then

$$
\begin{aligned}
\left\|\psi_{n_{k}}-\psi_{n_{l}}\right\| & \leq\left\|\bar{\varphi} \psi_{n_{k}}-\bar{\varphi} \psi_{n_{l}}\right\|+\left\|(1-\bar{\varphi}) \psi_{n_{k}}\right\|+\left\|(1-\bar{\varphi}) \psi_{n_{l}}\right\| \\
& \leq \frac{\epsilon}{2}+\frac{\epsilon}{4}+\frac{\epsilon}{4} \leq \epsilon
\end{aligned}
$$

as desired.

4.6 Corollary. $H=\triangle+V$ has compact resolvent if $M$ is compact and $V$ is continuous and symmetric.

\section{Basics of $L^{2}$-Cohomology and Hodge Decomposition In the Weighted Case}

We proceed now to reformulate parts of the Hodge theory of compact manifolds in our noncompact and weighted setting. Let

$$
\mathcal{H}_{\mu}^{p}=\left\{\omega \in L_{\mu}^{2} \Omega^{p}: \triangle_{\mu}^{p} \omega=0\right\}
$$

In order to connect $\mathcal{H}_{\mu}^{p}=\operatorname{ker} \triangle_{\mu}^{p}$ to the cohomology of the operator $d^{p}$, we need to carefully consider the Hilbert space structure on $p$-forms, assuming as usual that $M$ is complete.

5.1 Lemma. im $d^{p-1} \subseteq \operatorname{ker} d^{p}$ and $\operatorname{im}\left(d_{\mu}^{*}\right)^{p} \subseteq \operatorname{ker}\left(d_{\mu}^{*}\right)^{p-1}$.

This lemma addresses "domain issues" - that $d^{2}=0$ on $\Omega^{p-1}$ is fundamental. But in the $L_{\mu}^{2} \Omega^{\bullet}$ context with densely-defined closed operators $d$ and $d_{\mu}^{*}$, it contains an important idea: algebraic cancellation "overwhelms" apparent regularity difficulties in this case. (After all, $d$ is a first order differentiation operator. For an example of what should worry us, let $A=\frac{d}{d x}$ be the closed operator in $L^{2}[0,1]$ with $\mathcal{D}_{A}=\left\{f: f\right.$ absolutely continuous and $\left.f^{\prime} \in L^{2}[0,1]\right\}$. Then $\operatorname{im} A=L^{2}[0,1]$, so $\operatorname{im} A \nsubseteq \mathcal{D}_{A}$.)

Let

$$
L_{\mu}^{2} H^{p}=\frac{\operatorname{ker} d^{p}}{\operatorname{im} d^{p-1}}
$$


a definition justified by Lemma 5.1. The reader must keep in mind that the cohomology vector space $L_{\mu}^{2} H^{p}$ is dependent on the weight (measure) $d \mu$ because the domains of the closed operators $d^{p}$ are determined by $d \mu$.

An important domain issue is whether

$$
\triangle_{\mu}=d d_{\mu}^{*}+d_{\mu}^{*} d ?
$$

Recall $\triangle_{\mu}$ is the closure of $d \delta_{\mu}+\delta_{\mu} d$ on $\Omega_{c}^{\bullet}$, while on the right side we consider the closed operators $d, d_{\mu}^{*}$.

\subsection{Lemma.}

$$
\triangle_{\mu}=\left(D_{\mu}\right)^{2}=d d_{\mu}^{*}+d_{\mu}^{*} d
$$

i.e. with equality of domains.

Proof. For the first equality, note (by definition)

$$
\mathcal{D}_{\left(D_{\mu}\right)^{2}}=\left\{\omega \in \mathcal{D}_{D_{\mu}}: D_{\mu} \omega \in \mathcal{D}_{D_{\mu}}\right\} .
$$

Since $D_{\mu}$ is closed and self-adjoint, by $[\mathrm{ReS}], \mathrm{X} .25,\left(D_{\mu}\right)^{2}=D_{\mu}^{*} D_{\mu}$ is self-adjoint. In particular, $\left(D_{\mu}\right)^{2}$ is closed and is an extension of $d \delta_{\mu}+\delta_{\mu} d$ on $\Omega_{c}^{\bullet}$.

But we have proved that $d \delta_{\mu}+\delta_{\mu} d$ is essentially self-adjoint (Theorem 4.3), and $\triangle_{\mu}$ is its (self-adjoint) closure. Since a proper closed extension of a self-adjoint operator cannot be self-adjoint, this shows

$$
\triangle_{\mu}=\left(D_{\mu}\right)^{2} \text {. }
$$

That $\left(D_{\mu}\right)^{2}=d d_{\mu}^{*}+d_{\mu}^{*} d$ follows from the facts that $D_{\mu}=d+d_{\mu}^{*}, d^{2}=0$, and $\left(d_{\mu}^{*}\right)^{2}=0$. By definition

$$
\mathcal{D}_{d d_{\mu}^{*}+d_{\mu}^{*} d}=\left\{\omega \in \mathcal{D}_{d_{\mu}^{*}} \cap \mathcal{D}_{d}: d \omega \in \mathcal{D}_{d_{\mu}^{*}} \text { and } d_{\mu}^{*} \omega \in \mathcal{D}_{d}\right\},
$$

and we see that this is the same domain as $\mathcal{D}_{\left(D_{\mu}\right)^{2}}$ above.

The following is the standard regularity result for elliptic operators, phrased for our uses:

\subsection{Theorem.}

$$
\bigcap_{n=1}^{\infty} \mathcal{D}_{\left(\triangle_{\mu}\right)^{n}} \subset \Omega^{\bullet}
$$

We assert the following: (5.1) is well known if $d \mu=d x$, i.e. for the ordinary Hodge Laplacian; (5.1) is really local, i.e. if $U \subset M$ is a bounded open submanifold, and if $\alpha \in \bigcap_{n} \mathcal{D}_{\left(\triangle_{\mu}\right)^{n}}$ is supported in $U$, then $\alpha \in \Omega^{\bullet}(U)$; and finally, only the highest order part of $\triangle_{\mu}$ matters, i.e. since $\triangle$ is of second order but $L_{X}$ is only of first order, $\triangle_{\mu}=\triangle-2 L_{\nabla h}$ has the same elliptic regularity as $\triangle$. The reader can find these results in [Wa], [Ga], or [deR].

As a consequence of Theorem 5.3, if $\alpha \in L_{\mu}^{2} \Omega^{\bullet}$ and $\beta \in \Omega_{c}^{\bullet}$, and if for every $\theta \in \Omega_{c}^{\bullet}$,

$$
\left\langle\triangle_{\mu} \theta, \alpha\right\rangle_{\mu}=\langle\theta, \beta\rangle_{\mu},
$$

then in fact $\alpha \in \Omega^{\bullet} \cap \mathcal{D}_{\triangle_{\mu}}$ and $\triangle_{\mu} \alpha=\beta$. That is, (5.2) implies $\alpha \in \mathcal{D}_{\left(\triangle_{\mu}\right)^{*}}$, but since $\triangle_{\mu}$ is self-adjoint, we have $\alpha \in \mathcal{D}_{\triangle_{\mu}}$ and $\triangle_{\mu} \alpha=\beta$. But since $\beta \in \mathcal{D}_{\left(\triangle_{\mu}\right)^{n}}$ for any $n$, we conclude $\alpha \in \bigcap_{n} \mathcal{D}_{\left(\triangle_{\mu}\right)^{n}}$ as well.

5.4 Corollary. $\mathcal{H}_{\mu}^{p} \subseteq \Omega^{p}$, i.e. $\mu$-harmonic forms are smooth. 


\subsection{Theorem.}

$$
\mathcal{H}_{\mu}^{p}=\left\{\omega \in \mathcal{D}_{d^{p}} \cap \mathcal{D}_{\left(d_{\mu}^{*}\right)^{p}} \subset L_{\mu}^{2} \Omega^{p}: d \omega=0 \text { and } d_{\mu}^{*} \omega=0\right\} .
$$

Proof. Suppose $d \omega=d_{\mu}^{*} \omega=0$. Let $\theta \in \Omega_{c}^{p}$. Then

$$
\left\langle\triangle_{\mu}^{p} \theta, \omega\right\rangle_{\mu}=\left\langle d_{\mu}^{*} d \theta, \omega\right\rangle_{\mu}+\left\langle d d_{\mu}^{*} \theta, \omega\right\rangle_{\mu}=\langle d \theta, d \omega\rangle_{\mu}+\left\langle d_{\mu}^{*} \theta, d_{\mu}^{*} \omega\right\rangle_{\mu}=0 .
$$

As above, $\triangle_{\mu}^{p} \omega=0$, i.e. $\omega \in \mathcal{H}_{\mu}^{p}$.

Let $\phi \in \mathcal{H}_{\mu}^{p}$. Since we have been careful with domain issues above, the computation

$$
0 \leq\langle d \phi, d \phi\rangle_{\mu}+\left\langle d_{\mu}^{*} \phi, d_{\mu}^{*} \phi\right\rangle_{\mu}=\left\langle\left(d_{\mu}^{*} d+d d_{\mu}^{*}\right) \phi, \phi\right\rangle_{\mu}=\left\langle\triangle_{\mu} \phi, \phi\right\rangle_{\mu}=0
$$

shows $d \phi=d_{\mu}^{*} \phi=0$, since $\phi \in \mathcal{D}_{\triangle \mu}$ implies $\phi \in \mathcal{D}_{d d_{\mu}^{*}+d_{\mu}^{*} d} \subset \mathcal{D}_{d_{\mu}^{*}} \cap \mathcal{D}_{d}$. (A "constructive" derivation of this result is the theorem of A. Andreotti and E. Vesentinisee $[\mathrm{AV}]$ or $[\mathrm{deR}]$ - which was itself one of the first theorems addressing the noncompact case.)

5.6 Corollary. There is a natural injection $j_{p}: \mathcal{H}_{\mu}^{p} \rightarrow L_{\mu}^{2} H^{p}$, given by $\omega \mapsto[\omega]$.

Proof. By the above, $\omega \in \mathcal{H}_{\mu}^{p}$ implies $d \omega=0$, so $j_{p}$ makes sense. Suppose $[\omega]=0$ in $L_{\mu}^{2} H^{p}$, so $\omega=d \phi$ for some $\phi \in \mathcal{D}_{d}$. Then $0=\left\langle d_{\mu}^{*} \omega, \phi\right\rangle_{\mu}=\langle d \phi, d \phi\rangle_{\mu}=\|\omega\|^{2}$, so $\omega=0$. Thus $j_{p}$ is injective, as claimed.

The decomposition theorem below is a weak decomposition in the sense that the closure of the image of $d^{p-1}$ is taken in the decomposition. We shall see that this is a major issue.

5.7 Theorem (Classical).

$$
\begin{aligned}
L_{\mu}^{2} \Omega^{p} & =\overline{\mathrm{im} \triangle_{\mu}^{p}} \oplus \mathcal{H}_{\mu}^{p} \\
& =\overline{\mathrm{im}\left(d_{\mu}^{*}\right)^{p+1}} \oplus \overline{\mathrm{im} d^{p-1}} \oplus \mathcal{H}_{\mu}^{p} \\
& =\left(\operatorname{ker} d^{p}\right)^{\perp} \oplus \overline{\mathrm{im} d^{p-1}} \oplus \mathcal{H}_{\mu}^{p} .
\end{aligned}
$$

Proof. Since $\triangle_{\mu}^{p}$ and $d^{p}$ are closed, so

$$
\left(\operatorname{ker} \triangle_{\mu}^{p}\right)^{\perp}=\overline{\operatorname{im} \triangle_{\mu}^{p}} \quad \text { and } \quad\left(\operatorname{ker} d^{p}\right)^{\perp}=\overline{\operatorname{im}\left(d_{\mu}^{*}\right)^{p+1}}
$$

we need only prove

$$
\overline{\mathrm{im} \triangle_{\mu}^{p}}=\overline{\overline{\mathrm{im}\left(d_{\mu}^{*}\right)^{p+1}}} \oplus \overline{\mathrm{im} d^{p-1}} .
$$

The " $\subseteq$ " direction of (5.3) follows from Lemma 5.2.

Suppose $\alpha \in \overline{\operatorname{im}\left(d_{\mu}^{*}\right)^{p+1}} \oplus \overline{\operatorname{im} d^{p-1}}$, so $\alpha=\lim _{n} d_{\mu}^{*} \alpha_{n}+\lim _{n} d \beta_{n}$ for $\alpha_{n} \in \Omega_{c}^{p+1}$ and $\beta_{n} \in \Omega_{c}^{p-1}$. Let $\phi \in \mathcal{H}_{\mu}^{p}$, so by Corollary 5.4 and Theorem 5.5, $\phi \in \mathcal{D}_{d} \cap \mathcal{D}_{d_{\mu}^{*}} \cap \Omega^{p}$ as well. Thus

$$
\langle\alpha, \phi\rangle_{\mu}=\lim _{n}\left\langle\alpha_{n}, d \phi\right\rangle_{\mu}+\lim _{n}\left\langle\beta_{n}, d_{\mu}^{*} \phi\right\rangle_{\mu}=0
$$

by Theorem 5.5. So $\alpha \in\left(\mathcal{H}_{\mu}^{p}\right)^{\perp}=\overline{\mathrm{im} \triangle_{\mu}^{p}}$, yielding (5.3).

5.8 Corollary. $\mathcal{H}_{\mu}^{p} \cong\left(\operatorname{ker} d^{p}\right) /\left(\overline{\operatorname{im} d^{p-1}}\right)$.

The following is logically just a corollary to Theorem 5.7 but summarizes the main issue in the noncompact case. 
5.9 Theorem. $\mathcal{H}_{\mu}^{p} \cong L_{\mu}^{2} H^{p}$ iff im $d^{p-1}$ is closed, and in that case the isomorphism is given by $j_{p}$.

Next, we address a condition on $\triangle_{\mu}$ under which the isomorphism of Theorem 5.9 is valid.

We say a self-adjoint operator $A$ has a spectral gap if either 0 is an isolated point of the spectrum $\sigma(A)$ or $0 \notin \sigma(A)$. The existence of a spectral gap of a self-adjoint operator $A$ is equivalent to the condition that the image of $A$ is closed.

5.10 Theorem (Also classical). If $\triangle_{\mu}^{p}$ in $L_{\mu}^{2} \Omega^{p}$ has a spectral gap, then

$$
L_{\mu}^{2} \Omega^{p}=\operatorname{im}\left(d_{\mu}^{*}\right)^{p+1} \oplus \operatorname{im} d^{p-1} \oplus \operatorname{ker} \triangle_{\mu}^{p},
$$

and in particular,

$$
\mathcal{H}_{\mu}^{p} \cong L_{\mu}^{2} H^{p}
$$

Proof. Suppose $\inf \sigma\left(\triangle_{\mu}\right) \backslash\{0\}=\epsilon>0$. Let $\rho(x)=\frac{1}{x}$ for $x \geq \epsilon$ and $\rho(x)=0$ for $0 \leq x<\epsilon$. Define the bounded operator $\rho\left(\triangle_{\mu}\right)$ by the spectral theorem. Also, by the spectral theorem $\operatorname{im} \rho\left(\triangle_{\mu}\right) \subseteq \mathcal{D}_{\triangle_{\mu}}$, so $\triangle_{\mu} \rho\left(\triangle_{\mu}\right)$ is everywhere defined. In fact, $\rho\left(\triangle_{\mu}\right)$ is "almost the inverse" of $\triangle_{\mu}$, in the sense that

$$
I-\triangle_{\mu} \rho\left(\triangle_{\mu}\right)=\Pi,
$$

where $\Pi$ is the projection of $L_{\mu}^{2} \Omega^{p}$ onto ker $\triangle_{\mu}$.

In particular,

$$
L_{\mu}^{2} \Omega^{p}=\operatorname{im}\left[\triangle_{\mu} \rho\left(\triangle_{\mu}\right)\right] \oplus \operatorname{ker} \triangle_{\mu} .
$$

By Lemma 5.2, $\triangle_{\mu}=d_{\mu}^{*} d+d d_{\mu}^{*}$ (i.e. including equality of domains), so

$$
\triangle_{\mu} \rho\left(\triangle_{\mu}\right) \omega=d_{\mu}^{*} d \rho\left(\triangle_{\mu}\right) \omega+d d_{\mu}^{*} \rho\left(\triangle_{\mu}\right) \omega,
$$

thus $\operatorname{im}\left[\triangle_{\mu} \rho\left(\triangle_{\mu}\right)\right] \subseteq \operatorname{im} d_{\mu}^{*} \oplus \operatorname{im} d$. Note that $\operatorname{im} d_{\mu}^{*}$, im $d$, and ker $\triangle_{\mu}$ are mutually orthogonal and so we are done.

Recalling that $\triangle_{\mu}$ is elliptic, we see that

5.11 Corollary (Classical). If $\triangle_{\mu}$ has a spectral gap on $L_{\mu}^{2} \Omega^{\bullet}$, then since $\triangle_{\mu}$ is also elliptic in the sense of Theorem 5.3, we have

$$
\mathcal{H}_{\mu}^{p} \cong L_{\mu}^{2} H^{p} \cong \frac{\left.\operatorname{ker} d\right|_{\Omega^{p} \cap \mathcal{D}_{d^{p}}}}{\left.\operatorname{im} d\right|_{\Omega^{p-1} \cap \mathcal{D}_{d^{p-1}}}} .
$$

The smoothed $L_{\mu}^{2}$-cohomology group on the right in Corollary 5.11 is not, in general, isomorphic to $H_{\mathrm{de}}^{p} \mathrm{Rham}(M)$ or $H_{\mathrm{de} \mathrm{R}, c}^{p}(M)$. The smoothed group still involves the quotient of forms with controlled growth specified by $d \mu$.

Now, in the case where $M$ is compact, and for $\mu$ given by a nowhere-vanishing density with respect to $d x, \triangle_{\mu}^{p}$ has a spectral gap. Actually, a much stronger condition holds if $M$ is compact: $\triangle_{\mu}^{p}$ has compact resolvent, by Corollary 4.6. In particular, $\triangle^{p}$ has compact resolvent, and that fact, along with elliptic regularity, are the two pillars of the classical proof of the Hodge theorem. If $\triangle_{\mu}^{p}$ has compact resolvent, then $\mathcal{H}_{\mu}^{p}=\operatorname{ker} \triangle_{\mu}^{p}$ has finite dimension, a conclusion which does not follow from $\triangle_{\mu}^{p}$ having a spectral gap.

Furthermore, in the compact case,

$$
\Omega^{\bullet}(M) \subset L_{\mu}^{2} \Omega^{\bullet} \text { for any measure } d \mu=e^{2 h} d x .
$$


We conclude that the Hodge theorem holds with "full strength" for $\triangle_{\mu}$ on compact $M$.

5.12 Theorem. If $M^{n}$ is compact, orientable, and Riemannian, and if $d \mu=e^{2 h} d x$ for $h \in C^{\infty}(M)$, then

$$
\Omega^{p}(M)=\mathcal{H}_{\mu}^{p} \oplus \operatorname{im} d^{p-1} \oplus \operatorname{im} \delta_{\mu}^{p+1}
$$

is orthogonal with respect to $\langle\cdot, \cdot\rangle_{\mu}$, and in particular

$$
\mathcal{H}_{\mu}^{p} \cong H_{\text {de Rham }}^{p}(M) .
$$

Theorem 5.12 can be used to prove that the cohomology of a smooth compact manifold is of finite rank, since in fact $\triangle_{\mu}$ has compact resolvent.

\section{The Heat Kernel Weighted Laplacian ON 0-FORMS AND $n$-FORMS}

In this section we take $d \mu$ to be a heat kernel measure

$$
d \mu=\rho_{t}\left(x_{0}, x\right) d x
$$

for fixed $x_{0} \in M$ and $t>0$.

When using a heat kernel as a density for a measure, it is natural to consider complete manifolds with Ricci curvature bounded below, because in that case a heat kernel is unique and has integral one ([Da1], Theorem 5.2.6). That is, a heat kernel measure $d \mu$ on $M$ is a probability measure if the Ricci curvature of $M$ is bounded below.

Theorem 6.1 below is a slight generalization to complete manifolds of a result which appears for compact manifolds in [DriH]. The original technique of proof is ascribed to D. Bakry and M. Ledoux in [DriH]. The extension here to complete manifolds is accomplished using the semigroup domination results of [DonL]. A stronger result than Theorem 6.1, namely the corresponding logarithmic Sobolev inequality, appears in $[\mathrm{Hs}]$.

6.1 Theorem. Let $M$ be connected and complete Riemannian. If $\operatorname{Ric}_{M} \geq-c$ for $c>0$ and $d \mu$ is a heat kernel measure, then for $k=\frac{1}{c}\left(e^{2 t c}-1\right)$ the inequality

$$
\|f\|_{\mu}^{2}-\left|\langle f, 1\rangle_{\mu}\right|^{2} \leq k\left\langle\triangle_{\mu}^{0} f, f\right\rangle_{\mu}
$$

holds for all $f \in \mathcal{D}_{\triangle_{\mu}^{0}}$. That is, $\triangle_{\mu}^{0}$ has a spectral gap and

$$
\mathcal{H}_{\mu}^{0}=\{\text { constant functions }\} .
$$

Proof. Let $f \in \Omega_{c}^{0}$. For $s>0$ define

$$
\left(P_{s} f\right)(y)=\int_{M} \rho_{s}(y, x) f(x) d x .
$$

Define $P_{0} f=f$. It is well-known that $P_{s} f \rightarrow f$ as $s \rightarrow 0, P_{s} P_{s^{\prime}} f=P_{s+s^{\prime}} f$, $P_{s} f \in \mathcal{D}_{\triangle^{0}}$, and $\frac{d}{d s}\left(P_{s} f\right)=-\triangle^{0} P_{s} f$.

We will prove the inequality

$$
\left|d P_{s} f\right| \leq e^{s c} P_{s}|d f|,
$$

where $s>0$ and $|\omega|_{x}^{2}=(\omega, \omega)_{x}$ if $\omega \in \Omega^{1}$. For the moment, assume (6.2).

Let $\phi: R \rightarrow R$ be a $C^{2}$ function such that $\phi^{\prime \prime} \geq 0$. Define, for $0 \leq s \leq t$,

$$
\psi(s)=P_{t-s} \phi\left(P_{s} f\right) .
$$


Note $\psi(0)=P_{t} \phi(f)$ and $\psi(t)=\phi\left(P_{t} f\right)$. A direct calculation shows

$$
\frac{d \psi}{d s}=-P_{t-s}\left(\phi^{\prime \prime}\left(P_{s} f\right)\left|d P_{s} f\right|^{2}\right) .
$$

Then by (6.2) and the Cauchy-Schwarz inequality,

$$
-\frac{d \psi}{d s} \leq P_{t-s}\left(\phi^{\prime \prime}\left(P_{s} f\right) e^{2 s c}\left(P_{s}|d f|\right)^{2}\right) \leq P_{t-s}\left(\phi^{\prime \prime}\left(P_{s} f\right) e^{2 s c} P_{s}\left(|d f|^{2}\right)\right) .
$$

Take $\phi(x)=x^{2}$. (The choice $\phi(x)=x \log x$ would essentially lead to a logarithmic Sobolev inequality.) Then $P_{t-s}\left(\phi^{\prime \prime}\left(P_{s} f\right) e^{2 s c} P_{s}\left(|d f|^{2}\right)\right)=2 e^{2 s c} P_{t}\left(|d f|^{2}\right)$ and

$$
P_{t}\left(f^{2}\right)-\left(P_{t} f\right)^{2}=-\int_{0}^{t} \frac{d \psi}{d s} d s \leq k P_{t}\left(|d f|^{2}\right) .
$$

Note (6.3) is an inequality between functions on $M$, but if we evaluate at $y=x_{0}$, we get $(6.1)$, since $P_{t}\left(f^{2}\right)\left(x_{0}\right)=\|f\|_{\mu}^{2}, P_{t} f\left(x_{0}\right)=\langle f, 1\rangle_{\mu}$, and $P_{t}\left(|d f|^{2}\right)\left(x_{0}\right)=$ $\|d f\|_{\mu}^{2}=\left\langle\triangle_{\mu}^{0} f, f\right\rangle_{\mu}$. Finally, note $\triangle_{\mu}^{0}$ is essentially self-adjoint on $\Omega_{c}^{0}$, so (6.1) applies to any $f \in \mathcal{D}_{\triangle_{\mu}^{0}}$.

Now we prove (6.2). We will need to consider heat kernels on domains in $M$. All the required facts appear in Lemma 3 and Theorem 4 of Section VIII.2 of [Cha1].

Let $Q$ be a regular domain in $M$. Let $\rho_{s, Q}(x, y)$ be the heat kernel associated to the Dirichlet problem on $Q$. Consider also the integral kernel $K_{s, Q}^{1}(x, y) \in$ $\operatorname{End}\left(T_{y}^{*} M, T_{x}^{*} M\right)$ associated to the Dirichlet problem on $Q$ for the (ordinary) Hodge Laplacian $\triangle^{1}$ on one-forms.

H. Donnelly and P. Li ([DonL], Theorem 4.3) show that if $\operatorname{Ric}_{M} \geq-c$, then

$$
\left\|K_{s, Q}^{1}(x, y)\right\| \leq e^{s c} \rho_{s, Q}(x, y),
$$

where $\|\cdot\|$ is the norm defined with respect to $(\cdot, \cdot)_{x}$ on $T_{x}^{*} M$.

Because $d \triangle^{0}=\triangle^{1} d$,

$$
\left|d P_{s, Q} g\right|=\left|P_{s, Q}^{1}(d g)\right| \leq e^{s c} P_{s, Q}|d g|,
$$

where $g \in \Omega_{c}^{0}(Q),\left(P_{s, Q} g\right)(y)=\int_{Q} \rho_{s, Q}(y, x) g(x) d x$, and for $\omega \in \Omega_{c}^{1}(Q),\left(P_{s, Q}^{1} \omega\right)(y)$ $=\int_{Q} K_{s, Q}^{1}(y, x) \omega(x) d x$.

Let $\left\{Q_{j}\right\}$ be an increasing exhaustion of $M$ by regular domains. We find in [Cha1] that $\rho_{s, Q_{j}} \nearrow \rho_{s}$, and the convergence is uniform on compact subsets of $M$ for derivatives of all orders. Noting that the support of $f$ is compact, we see

$$
d P_{s, Q_{j}} f \rightarrow d P_{s} f \quad \text { and } \quad P_{s, Q_{j}}|d f| \rightarrow P_{s}|d f|,
$$

so $\left|d P_{s} f\right| \leq e^{s c} P_{s}|d f|$ by $(6.4)$, as desired.

By Theorem 5.10, $L_{\mu}^{2} \Omega^{0}=\mathcal{H}_{\mu}^{0} \oplus \operatorname{im}\left(d_{\mu}^{*}\right)^{1}$ if $\triangle_{\mu}^{0}$ has a spectral gap.

If Theorem 6.1 applies, then as $t \searrow 0$ the spectral gap of $\triangle_{\mu}^{0}$ grows like $\frac{1}{2 t}$.

Theorem 6.1 has a simple topological interpretation. Since we assumed $M$ connected, we have

$$
\operatorname{dim} \mathcal{H}_{\mu}^{0}=1=b_{0}=\operatorname{dim} H_{\mathrm{de} \operatorname{Rham}}^{0}(M) .
$$

Obvious modifications of our methods show $\operatorname{dim} \mathcal{H}_{\mu}^{0}=b_{0}=(\#$ of connected components of $M)$ if $M$ is not connected. In that case, choose one basepoint $x_{0}^{\alpha}$ per 
component $M^{\alpha}$ and work in

$$
L_{\mu}^{2} \Omega^{\bullet}=\bigoplus_{\alpha} L_{\rho_{t}\left(x_{0}^{\alpha}, x\right) d x}^{2} \Omega^{\bullet} .
$$

The proof of Theorem 6.1 depends on the fact that $\triangle_{\mu}^{0}$ is a Dirichlet form operator:

$$
\left\langle\triangle_{\mu}^{0} f, f\right\rangle_{\mu}=\int_{M}(d f, d f)_{x} \rho_{t}\left(x, x_{0}\right) d x \quad \text { for all } \quad f \in \mathcal{D}_{\triangle_{\mu}^{0}}
$$

Since $\triangle_{\mu}^{n}$ is unitarily equivalent to $\triangle_{\mu^{-}}^{0}$ (by Theorem 3.1), and since the latter is also a Dirichlet form operator, we may identify the kernel $\mathcal{H}_{\mu}^{n}$ of $\triangle_{\mu}^{n}$ acting on $n$-forms.

6.2 Theorem. If $\alpha=\int_{M} \frac{1}{\rho_{t}\left(x, x_{0}\right)} d x$, then

$$
\operatorname{dim} \mathcal{H}_{\mu}^{n}= \begin{cases}1, & \alpha<+\infty \\ 0, & \alpha=+\infty\end{cases}
$$

If $\alpha<+\infty$ then $\mathcal{H}_{\mu}^{n}$ is spanned by

$$
\tilde{\omega}=\left[\rho_{t}\left(\cdot, x_{0}\right)\right]^{-1} \star 1 .
$$

Proof. By Theorem 3.1, $\omega \in \operatorname{ker} \triangle_{\mu}^{n}$ iff $\xi=e^{2 h} \star \omega \in \operatorname{ker} \triangle_{\mu^{-}}^{0}$. If $\xi \in \operatorname{ker} \triangle_{\mu^{-}}^{0}$ then

$$
0=\left\langle\triangle_{\mu^{-}}^{0} \xi, \xi\right\rangle_{\mu^{-}}=\int_{M}(d \xi, d \xi)_{x} \mu^{-}(d x)=\int_{M}(d \xi, d \xi)_{x} \frac{1}{\rho_{t}\left(x, x_{0}\right)} d x
$$

so $\xi$ is constant. But $1 \in L_{\mu^{-}}^{2} \Omega^{0}$ iff $\int_{M} \frac{1}{\rho_{t}\left(x, x_{0}\right)} d x<+\infty$. If $1 \in L_{\mu^{-}}^{2} \Omega^{0}$ then $\omega=e^{-2 h} \star 1$ spans $\operatorname{ker} \triangle_{\mu}^{n}$.

It is possible - though we know of no examples - that $\alpha<+\infty$ for some noncompact and complete $M$. Confirmation of the following conjecture would be evidence that the condition " $\mathrm{Ric}_{M} \geq-c$ " is indeed a natural geometric condition in our context.

6.3 Conjecture. If $\operatorname{Ric}_{M} \geq-c$ then

$$
\alpha=\int_{M} \frac{1}{\rho_{t}\left(x, x_{0}\right)} d x<+\infty \quad \text { iff } \quad M \text { is compact. }
$$

Under a stronger geometric restriction, we may verify this immediately.

6.4 Theorem. If $\operatorname{Ric}_{M} \geq 0$ then

$$
\alpha<+\infty \text { iff } M \text { is compact, }
$$

and thus

$$
\mathcal{H}_{\mu}^{n} \cong H_{\text {de Rham }}^{n}(M)
$$

Proof. If $M$ is complete and Ric $\geq 0$, then vol $M<+\infty$ iff $M$ is compact ([Cha2], p. 150). If $M$ is compact, then $\rho$ is bounded below, so $\alpha<+\infty$, but if $\operatorname{vol} M=+\infty$, then $\alpha=+\infty$. 
Note that the $n$th cohomology of an orientable manifold $M$ is easily found by Poincaré duality (Theorem 3.3):

$$
\begin{aligned}
H_{\mathrm{de} \mathrm{Rham}}^{n}(M) & \cong H_{\mathrm{de} \mathrm{R,c}}^{0}(M) \\
& =\left\{f \in C_{c}^{\infty}(M): d f=0\right\} \\
& =\{\text { compactly supported constant functions }\} \\
& \cong\left\{\begin{array}{lll}
R, & M & \text { compact } \\
0, & M & \text { not compact. }
\end{array}\right.
\end{aligned}
$$

It would seem that in order to prove a spectral gap for $\triangle_{\mu}^{p}$ via an inequality along the lines of Theorem 6.1, we are obliged to identify the kernel $\mathcal{H}_{\mu}^{p}$ in advance. We expect such a gap to exist, but this may be more than can be asked in proving a Hodge theorem.

\section{Heat Kernels and "Hodge Theory" on Euclidean Spaces}

In Euclidean space $R^{n}$ we may calculate explicitly the heat kernel weighted Laplacian $\triangle_{\mu}$, as well as the inverse heat kernel weighted Laplacian $\triangle_{\mu^{-}}$. Recall that $\rho_{t}(x, y)=(4 \pi t)^{-n / 2} \exp \left((x-y)^{2} / 4 t\right)$. Choose $x_{0}=0$ and $t=\frac{1}{2}$. We ignore the overall constant $(2 \pi)^{-n / 2}$.

7.1 Theorem. Let $d \mu=e^{-|x|^{2} / 2} d x$ and let $d \mu^{-}=e^{+|x|^{2} / 2} d x$. Define $\triangle_{\mu}^{p}$ and $\triangle_{\mu^{-}}^{p}$ in $L_{\mu}^{2} \Omega^{p}\left(R^{n}\right)$ and $L_{\mu^{-}}^{2} \Omega^{p}\left(R^{n}\right)$, respectively. Then

$$
\operatorname{ker} \triangle_{\mu}^{p} \cong \begin{cases}R, & p=0 \\ 0, & p>0\end{cases}
$$

and

$$
\operatorname{ker} \triangle_{\mu^{-}}^{p} \cong \begin{cases}0, & p<n, \\ R, & p=n .\end{cases}
$$

Both $\triangle_{\mu}^{p}$ and $\triangle_{\mu^{-}}^{p}$ have compact resolvent, for all $p$.

The calculation is straightforward, given the following lemma.

7.2 Lemma. Let $\omega \in \Omega_{c}^{p}$. Using multi-index notation and the summation convention, we may write $\omega$ globally as $\omega=f_{I} d x^{I}$. Then

$$
\triangle_{\mu}^{p} \omega=\left(-\frac{\partial^{2} f_{I}}{\left(\partial x^{i}\right)^{2}}+x^{i} \frac{\partial f_{I}}{\partial x^{i}}+p f_{I}\right) d x^{I} .
$$

Furthermore, the spectrum of

$$
\triangle_{\mu}^{0}=-\frac{\partial^{2}}{\left(\partial x^{i}\right)^{2}}+x^{i} \frac{\partial}{\partial x^{i}} \quad \text { acting in } \quad L_{\mu}^{2} \Omega^{0}\left(R^{n}\right)
$$

consists precisely of finite-multiplicity eigenvalues $\{0,1,2, \ldots\}$, and $\operatorname{ker} \triangle_{\mu}^{0}=$ \{constants\}. 
Proof. Recall that $\triangle_{\mu}=\triangle-2 L_{\nabla h}$ on $L_{\mu}^{2} \Omega^{\bullet}$ if $d \mu=e^{2 h} d x$. Let $h(x)=-\frac{|x|^{2}}{4}$, so $\nabla h=-\sum_{i=1}^{n} \frac{x^{i}}{2} \frac{\partial}{\partial x^{i}}$. Then

$$
\begin{aligned}
2 L_{\nabla h}\left(f_{I} d x^{I}\right) & =d i_{-x^{i} \frac{\partial}{\partial x^{i}}}\left(f_{I} d x^{I}\right)+i_{-x^{i} \frac{\partial}{\partial x^{i}}} d\left(f_{I} d x^{I}\right) \\
& =-d\left(x^{i} f_{I}\right) \wedge i \frac{\partial}{\partial x^{i}} d x^{I}-x^{i} i \frac{\partial}{\partial x^{i}} d f_{I} \wedge d x^{I} \\
& =-\left[f_{I} d x^{i} \wedge i \frac{\partial}{\partial x^{i}}+x^{i} \frac{\partial f_{I}}{\partial x^{j}}\left(d x^{j} \wedge i \frac{\partial}{\partial x^{i}}+i \frac{\partial}{\partial x^{i}} d x^{j} \wedge\right)\right] d x^{I} .
\end{aligned}
$$

Two identities

$$
\begin{gathered}
d x^{j} \wedge i_{\frac{\partial}{\partial x^{i}}}+i_{\frac{\partial}{\partial x^{i}}} d x^{j} \wedge=\delta_{i}^{j} \quad \text { on } \quad \wedge^{\bullet} T_{x}^{*} M \\
\sum_{i=1}^{n} d x^{i} \wedge i_{\frac{\partial}{\partial x^{i}}}=p \quad \text { on } \quad \wedge^{p} T_{x}^{*} M
\end{gathered}
$$

pertain, giving

$$
2 L_{\nabla h}\left(f_{I} d x^{I}\right)=\left(-p f_{I}-x^{i} \frac{\partial f_{I}}{\partial x^{i}}\right) d x^{I} .
$$

Equation (7.3) follows.

Let $L=-\frac{\partial^{2}}{\partial x^{2}}+x \frac{\partial}{\partial x}$ as an operator in $L_{\mu}^{2}\left(R^{1}\right)$. This operator has a well-known complete orthogonal system of eigenfunctions, namely, the Hermite polynomials

$$
\left\{H_{j}(x)\right\}_{j=0}^{\infty},
$$

$H_{0}(x)=1, H_{1}(x)=x, H_{2}(x)=x^{2}-1$, etc., with

$$
L H_{j}=j H_{j} \text {. }
$$

Thus $\triangle_{\mu}^{0}$ has a complete orthogonal system of eigenfunctions given by "tensor products"

$$
\left\{H_{j_{1}}\left(x^{1}\right) \ldots H_{j_{n}}\left(x^{n}\right)\right\}_{j_{1}, \ldots, j_{n}=0}^{\infty},
$$

and so $\triangle_{\mu}^{0}$ has spectrum $\{0,1,2, \ldots\}$, with ker $\triangle_{\mu}^{0} \equiv\{$ constants $\}$.

Proof of Theorem 7.1. On the one hand,

$$
\triangle_{\mu}^{p}\left(f_{I} d x^{I}\right)=\left(\triangle_{\mu}^{0} f_{I}+p f_{I}\right) d x^{I}
$$

but also $\triangle_{\mu}^{0}=\delta_{\mu} d$ is positive on $L_{\mu}^{2}\left(R^{n}\right)$; thus

$$
\operatorname{ker} \triangle_{\mu}^{p}=0 \text { for } \quad p>0 .
$$

It is easy to check that $\left(f \in C^{2}\right)$

$$
\left(-\frac{\partial^{2}}{\partial x^{2}}-x \frac{\partial}{\partial x}\right)\left(f e^{-\frac{x^{2}}{2}}\right)=\left[\left(-\frac{\partial^{2}}{\partial x^{2}}+x \frac{\partial}{\partial x}\right) f\right] e^{-\frac{x^{2}}{2}}+1 \cdot f e^{-\frac{x^{2}}{2}}
$$

on $R^{1}$ and hence that (see also Theorem 3.1)

$$
\triangle_{\mu^{-}}^{p}\left(e^{-\frac{|x|^{2}}{2}} f_{I} d x^{I}\right)=e^{-\frac{|x|^{2}}{2}}\left(\triangle_{\mu}^{0} f_{I}+(n-p) f_{I}\right) d x^{I}
$$

on $R^{n}$. Since $\triangle_{\mu}^{0}$ is positive, this implies that $\operatorname{ker} \triangle_{\mu^{-}}^{p}=0$ for $p<n$ and $\operatorname{ker} \triangle_{\mu^{-}}^{n} \cong$ $R$ as claimed.

Since $\triangle_{\mu}^{0}$ has compact resolvent, it follows that both $\triangle_{\mu}^{p}$ and $\triangle_{\mu^{-}}^{p}$ have compact resolvent. 
In summary,

$$
H_{\text {de Rham }}^{p}\left(R^{n}\right) \cong \operatorname{ker} \triangle_{\mu}^{p}, \quad 0 \leq p \leq n,
$$

and

$$
H_{\mathrm{de} \mathrm{R,c}}^{p}\left(R^{n}\right) \cong \operatorname{ker} \triangle_{\mu^{-}}^{p}, \quad 0 \leq p \leq n,
$$

which we take as a "Hodge theorem for $R^{n}$."

Since we may prove (7.1) from (7.2) or vice versa by using Theorem 3.1,

$$
\star_{\mu} \triangle_{\mu}^{p}=\triangle_{\mu^{-}}^{n-p} \star_{\mu},
$$

we say that $\triangle_{\mu}$ gives a "Hodge theory for $R^{n}$, compatible with Poincaré duality on $R^{n}$."

The real intent of this section is motivation - so we ask a final question: What happens if $n=\infty$ ?

The answer is an analytical can of worms, of course. However, note the essential heuristic about infinite-dimensional manifolds which are also measure spaces: there is no Riemann-Lebesgue measure, but there may be a well-defined Gaussian measure. Thus we look for a "Hodge theory" on infinite-dimensional manifolds based on an abstract Wiener space, which we take here as an " $n=\infty$ dimensional Euclidean space with a Gaussian measure." A Hodge-de Rham theory of infinite-dimensional manifolds is a current goal among some analysts - see [P] and [Gro].

In the "Euclidean" case, I. Shigekawa proves the following theorem.

7.3 Theorem ([Sh]). Differential forms, the exterior derivative $d$, its adjoint $\delta_{B}$, and the Hodge Laplacian

$$
\triangle_{B}=d \delta_{B}+\delta_{B} d
$$

are defineable on an abstract Wiener space $B$, and in that case,

$$
\triangle_{B}^{p}=-L^{p}+p
$$

on p-forms, where $L^{p}$ is essentially the Ornstein-Uhlenbeck operator. It is known that $L$ is nonpositive, has spectral gap, and has kernel

$$
\operatorname{ker} L^{0}=\{\text { constant functions }\} .
$$

Thus we have a strong Hodge decomposition and

$$
\operatorname{ker} \triangle_{B}^{p} \cong \begin{cases}R, & p=0, \\ 0, & p>0 .\end{cases}
$$

The case of an abstract Wiener space corresponds to the measure $d \mu$ of this section. The measure $d \mu^{-}$, which has large density with respect to Lebesgue measure, naturally fails to exist if $n=\infty$.

\section{Compact Resolvent for the Heat Kernel Weighted LAPlaCian: SUfFicient CONDITIONS}

In Section 4 we showed that $\triangle_{\mu}$ was unitarily equivalent to a Schrödinger operator. That is, $\triangle_{\mu}^{U}=\triangle+V_{\mu}$ on $L^{2} \Omega^{\bullet}$, where

$$
V_{\mu}=|d h|^{2}+\sum_{i, j} H h\left(X_{i}, X_{j}\right)\left[a^{j}, a^{i *}\right] .
$$

On the other hand, in Section 6 we showed that, in reasonable generality, the heat kernel weighted Laplacian $\triangle_{\mu}$ has spectral gap when acting on functions. In 
Section 7 we showed that in Euclidean space this operator in fact has compact resolvent on forms of all degrees.

Using the form of $V_{\mu}$ above, we give in this section sufficient conditions on the density $e^{2 h}$ (for instance, on the heat kernel $\rho_{t}\left(x_{0}, \cdot\right)$ ) such that $\triangle_{\mu}^{U}$ has compact resolvent on the Hilbert space $L^{2} \Omega^{\bullet}$ of forms of all degrees. Our conditions take the form of asymptotic statements about the derivatives of $h$. Note that for the heat kernel density, $h(x)=\frac{1}{2} \ln \rho_{t}\left(x_{0}, x\right)$.

8.1 Theorem. Let $h \in C^{\infty}(M)$. Let

$$
\|H h\|_{x} \equiv \max _{Y \in T_{x} M}\left\{\frac{H h(Y, Y)}{|Y|^{2}}\right\},
$$

and let $\left\{X_{i}\right\}_{i=1}^{n}$ be an orthonormal basis of $T_{x} M$. If

$$
|d h(x)| \quad \text { goes to } \infty \text { at } \infty
$$

and if there exists $K \subset M$ compact and $c<1$ such that for all $x \in M \backslash K$,

$$
\|H h\|_{x} \leq \frac{c}{n}|d h(x)|^{2},
$$

then $V_{\mu}$ goes to $\infty$ at $\infty$, and thus

$$
\triangle_{\mu}^{U} \text { in } L^{2} \Omega^{\bullet} \text { has compact resolvent. }
$$

Proof. Note that on $\wedge^{\bullet} T_{x}^{*} M,\left\|\left[a^{j}, a^{i *}\right]\right\| \leq 1$. If $\alpha \in \wedge^{\bullet} T_{x}^{*} M$, then

$$
\left|\sum_{i, j} H h\left(X_{i}, X_{j}\right)\left[a^{j}, a^{i *}\right] \alpha\right| \leq \sum_{i, j}\left|H h\left(X_{i}, X_{j}\right)\right||\alpha| \leq n\|H h\|_{x}|\alpha| .
$$

If $(8.2)$ holds then

$$
\left|V_{\mu}(x) \alpha\right| \geq\left(|d h(x)|^{2}-n\|H h\|_{x}\right)|\alpha| \geq(1-c)|d h(x)|^{2}|\alpha|,
$$

so by $(8.1), V_{\mu}(x)$ goes to $\infty$ at $\infty$. Apply Theorem 4.5 to conclude $\triangle_{\mu}^{U}$ has compact resolvent.

Fix $t>0$ and consider the heat kernel measure $d \mu=\rho_{t}\left(x_{0}, x\right) d x$. At least heuristically,

$$
\rho_{t}\left(x_{0}, x\right) \quad \text { compares to } A e^{-B d\left(x_{0}, x\right)^{2}}
$$

for some constants $A, B>0$. It is therefore reasonable to expect

$$
|d h(x)| \sim d\left(x_{0}, x\right)
$$

and

$$
\|H h\|_{x} \text { is small compared to }|d h(x)|^{2} \text { for large } x \text {. }
$$

In the Euclidean case, $\|H h\|_{x}$ is constant and $|d h(x)|=c(t)\left|x-x_{0}\right|$.

The heuristic given in (8.3) has substantial confirmation in a large selection of heat kernel estimation theorems, for which [Da1] is perhaps the most accessible reference. We find there that if the Ricci curvature is nonnegative, we have the following actual situation. 
8.2 Theorem ([Da1], Theorems 5.5.11 and 5.6.1). If $\operatorname{Ric}_{M} \geq 0$, then

$$
(4 \pi t)^{-n / 2} \exp \left(\frac{-d\left(x_{0}, x\right)^{2}}{4 t}\right) \leq \rho_{t}\left(x_{0}, x\right)
$$

and for all $\delta>0$, there exists $a_{\delta}>0$ such that

$$
\rho_{t}\left(x_{0}, x\right) \leq a_{\delta}\left(\operatorname{vol} B\left(x_{0}, t^{1 / 2}\right)\right)^{-1} \exp \left(\frac{-d\left(x_{0}, x\right)^{2}}{4(1+\delta) t}\right) .
$$

These bounds do not hold if the Ricci curvature is merely bounded below, but the Gaussian character remains for many purposes (see [Da1]).

On the other hand, the "reasonable" heuristics (8.4) and (8.5), on the spatial derivatives of the logarithm of the heat kernel, are not well represented in the heat kernel literature. Note, however, that [Da2], [Gri], [St], and [MS] consider the derivatives of $\rho_{t}\left(x_{0}, \cdot\right)$ or $h$. Positive results include:

8.3 Theorem ([MS], (0.3); [MS], Corollary 2.29; and [St], (0.3)). Let $M$ be compact. Fix $x_{0} \in M$. Let $h_{t}(x)=\ln \rho_{t}\left(x_{0}, x\right)$. Then

$$
\lim _{t \searrow 0} t h_{t}(x)=\frac{-d\left(x_{0}, x\right)^{2}}{4}
$$

uniformly on $M$. Let $E(x)=\frac{1}{4} d\left(x_{0}, x\right)^{2}$. Let $C\left(x_{0}\right)$ be the cut locus of $x_{0}$. Then $E$ is smooth on $M \backslash C\left(x_{0}\right)$, and

$$
\begin{aligned}
\lim _{t \searrow 0} t d h_{t} & =-d E, \\
\lim _{t \searrow 0} t H h_{t} & =-H E
\end{aligned}
$$

uniformly on compact subsets of $M \backslash C\left(x_{0}\right)$.

Finally, there exists $c>0$ such that

$$
\max \left\{|d h(x)|^{2},\|H h\|_{H . S .}(x)\right\} \leq c\left(\frac{d\left(x_{0}, x\right)^{2}}{4 t^{2}}+\frac{1}{2 t}\right)
$$

for all $(t, x) \in\left(0, \frac{1}{2}\right] \times M$, where $\|\cdot\|_{H . S .}$ is the Hilbert-Schmidt norm on the finite dimensional Hilbert space $T_{x} M$. (Note that $\|A\| \leq\|A\|_{H . S .} \leq \sqrt{n}\|A\|$ if $A$ acts in the $n$-dimensional space $T_{x} M$.)

Thus, though it is reasonable to expect that the hypotheses of Theorem 8.1 apply to $h(x)=\frac{1}{2} \ln \rho_{t}\left(x_{0}, x\right)$ for a large class of noncompact and complete $M$, nevertheless proving this is a nontrivial application of modern "heat kernel estimates" technology. Specifically, we need to know that the first spatial derivatives of $h$ grow sufficiently fast, and we need to know that the second spatial derivatives of $h$ are in a certain sense small compared to the first derivatives.

It is worth recalling here that if the hypotheses of Theorem 8.1 were to apply to the heat kernel on some manifold $M$, then (by Theorem 5.10) $\mathcal{H}_{\mu}^{p} \cong L_{\mu}^{2} H^{p}$ for $0 \leq$ $p \leq n$. The topological significance of $\mathcal{H}_{\mu}^{p}$, in particular its relation to $H_{\mathrm{de}}^{p}$ Rham $(M)$, would remain undetermined.

$$
\begin{aligned}
& \text { 9. Weighted Laplacians for } d \mu=e^{ \pm r^{2}} d x \text { IN The } \\
& \text { Presence of a Distance Function } r(x)=d(x, S)
\end{aligned}
$$

Assume $M$ is connected, noncompact, orientable and complete.

In this section we show that if $M$ has a "good" distance function $r$-the precise meaning given below - then the weighted Hodge Laplacian $\triangle_{\mu}^{U}$ built from $d \mu=$ 
$\exp \left(c r^{2}\right) d x, c \neq 0$, has compact resolvent. Theorems 9.1 and 9.2 will show that certain manifolds with soul and pole (respectively) have just such an $r$.

Let us calculate the form of the weighted Laplacian $\triangle_{\mu}^{U}$, assuming the existence of a smooth $\left(C^{\infty}\right)$ distance function on $M \backslash S$

$$
r: x \mapsto d(x, S)
$$

for $S$ a compact subset of $M$.

Let $h(x)=\frac{c}{2} r^{2}$ for $c \in R$. For now we are interested in local calculations away from $S$, where $h$ is smooth. Letting $d \mu=e^{2 h} d x$, we have equation (4.4), which gives $\triangle_{\mu}^{U}=\triangle+V_{\mu}$. We will simplify the potential

$$
V_{\mu}=|d h|^{2}+\sum_{i, j=1}^{n} H h\left(X_{i}, X_{j}\right)\left[a^{j}, a^{i *}\right] .
$$

First, $|d r|=1$, so

$$
|d h|^{2}=c^{2} r^{2}
$$

Second,

$$
\begin{aligned}
H h(X, Y) & =\left(X Y-\nabla_{X} Y\right) h=c\left[X(r Y r)-r\left(\nabla_{X} Y r\right)\right] \\
& =c(X r)(Y r)+c r H r(X, Y),
\end{aligned}
$$

and since $(d r \otimes d r)(X, Y)=(X r)(Y r)$, we write

$$
H h=c d r \otimes d r+c r H r .
$$

Now,

$$
\begin{aligned}
H r(X, \nabla r) & =X \nabla r(r)-\nabla_{X} \nabla r(r)=X\langle\nabla r, \nabla r\rangle-\left\langle\nabla r, \nabla_{X} \nabla r\right\rangle \\
& =X(1)-\frac{1}{2} X\langle\nabla r, \nabla r\rangle=0,
\end{aligned}
$$

recalling $\nabla$ is the Levi-Civita connection. Thus we can choose an orthonormal basis $\left\{X_{1}=\nabla r, X_{2}, \ldots, X_{n}\right\}$ diagonalizing $d r \otimes d r$ and $H r$, giving

$$
\sum_{i, j} H h\left(X_{i}, X_{j}\right)\left[a^{j}, a^{i *}\right]=c\left[a^{1}, a^{1 *}\right]+c r \sum_{i=2}^{n} \operatorname{Hr}\left(X_{i}, X_{i}\right)\left[a^{i}, a^{i *}\right] .
$$

Let $\lambda_{i}=\operatorname{Hr}\left(X_{i}, X_{i}\right)$. Thus in local coordinates away from $S$,

$$
V_{\mu}=c^{2} r^{2}+c\left[a^{1}, a^{1 *}\right]+c r \sum_{i=2}^{n} \lambda_{i}\left[a^{i}, a^{i *}\right] .
$$

As an illustration, if $M=R^{n}$ with the Euclidean metric and $S$ is the origin, then $\lambda_{i}=1 / r$.

We are interested in finding $\triangle_{\mu}^{U}$ with spectral gap, or more specifically, compact resolvent. Recall $\triangle_{\mu}^{U}=\triangle+V_{\mu}$ has compact resolvent if $V_{\mu}$ goes to $\infty$ at $\infty$. Note $\left[a^{i}, a^{i *}\right]$ has norm 1 pointwise. Clearly, it would suffice to show

$$
\max _{i}\left|\lambda_{i}\right|=o(r) \quad \text { as } \quad r \rightarrow \infty
$$

to show $\triangle_{\mu}^{U}$ had compact resolvent. But we suspect that in many cases

$$
\max _{i}\left|\lambda_{i}\right|=O(1) \quad \text { as } \quad r \rightarrow \infty
$$


or even

$$
\max _{i}\left|\lambda_{i}\right|=O\left(\frac{1}{r}\right) \quad \text { as } \quad r \rightarrow \infty,
$$

in the case of nonnegative curvature, as suggested by the following.

Let $M$ be a noncompact, complete Riemannian manifold with nonnegative sectional curvatures. J. Cheeger and D. Gromoll ([CG], Theorem 2.2) have shown that $M$ must have the structure of a vector bundle over a compact submanifold. Specifically, such an $M$ contains a compact, totally convex submanifold $S$, with $\partial S=\emptyset$, such that $M$ is diffeomorphic to the normal bundle $N S$ of $S$. We say that $M$ has soul $S$. J. Escobar and A. Freire [EF1] suggest a further technical condition on $M \cong N S$, namely that

$$
\exp : N S \rightarrow M
$$

gives the diffeomorphism. In this case, it is known that $r(x)=d(x, S)$ is indeed smooth on $M \backslash S$ and that

$$
0 \leq \lambda_{i} \leq \frac{1}{r}
$$

9.1 Theorem. Let $M^{n}$ be a noncompact, oriented, complete Riemannian manifold with nonnegative sectional curvatures. Let $S$ be a soul of $M$, and assume exp : $N S \rightarrow M$ is a diffeomorphism. Let $r(x)=d(x, S)$ and let $h$ be a $C^{\infty}$ function on $M$ which equals $\frac{c}{2} r^{2}(c \neq 0)$ in the complement of a precompact neighborhood of $S$. If $d \mu=e^{2 h} d x$, then

$$
\triangle_{\mu}^{U} \text { acting in } L^{2} \Omega^{\bullet} \text { has compact resolvent. }
$$

Proof. By [EF1], Lemma 1.2, $r$ is smooth on $M \backslash S$, and

$$
0 \leq H r(X, X) \leq \frac{1}{r}|X|^{2},
$$

for any $X \in T_{x} M, x \in M \backslash S$.

Equation (9.1) holds, away from $S$. Since $0 \leq \lambda_{i}=\operatorname{Hr}\left(X_{i}, X_{i}\right) \leq \frac{1}{r}$, and $\left\|\left[a^{i}, a^{i *}\right]\right\|=1$ as an operator in the inner product space $\wedge^{\bullet} T_{x}^{*} M$ for any $x \in M$, we see

$$
V=c^{2} r^{2}+c\left[a^{1}, a^{1 *}\right]+c r \sum_{i=2}^{n} \lambda_{i}\left[a^{i}, a^{i *}\right] \geq c^{2} r^{2}-c n
$$

as a symmetric operator on $\wedge^{\bullet} T_{x}^{*} M$. As $c^{2} r^{2}-c n$ goes to $\infty$ at $\infty, \triangle_{\mu}^{U}$ has compact resolvent by Theorem 4.5.

A special case of a manifold with soul $S$ is that of a manifold with pole, i.e. for which there exists a point $x_{0} \in M$ such that

$$
\exp : T_{x_{0}} M \rightarrow M
$$

is a diffeomorphism. If $M$ possesses a pole, then $M$ is complete and diffeomorphic to $R^{n}$.

Theorem 9.1 applies to nonnegatively curved manifolds with pole. Note that we may reach the same conclusion on certain nonpositively curved manifolds with pole, as follows. 
9.2 Theorem. Let $M^{n}$ be a manifold with pole $x_{0}$. Let $r(x)=d\left(x, x_{0}\right)$ and let $h$ be a $C^{\infty}$ function on $M$ which equals $\frac{c}{2} r^{2}(c \neq 0)$ outside of $B_{\epsilon}\left(x_{0}\right)$, for some $\epsilon>0$. Let $d \mu=e^{2 h} d x$. Suppose the sectional curvatures of $M$ are nonpositive and bounded below; that is, suppose there exists $\beta>0$ such that for every $x \in M$ and every plane $\pi$ in $T_{x} M$ we have

$$
-\beta^{2} \leq K(\pi) \leq 0
$$

Then

$$
\max _{i}\left|\lambda_{i}\right|=O(1) \quad \text { as } \quad r \rightarrow \infty
$$

and therefore

$$
\triangle_{\mu}^{U} \text { in } L^{2} \Omega^{\bullet} \text { has compact resolvent. }
$$

Proof. We use the Hessian comparison theorem. We compare $M$ to the simply connected hyperbolic space of constant sectional curvature $N^{n}$ and to Euclidean space $R^{n}$.

Take $K_{N}=-\beta^{2}$. If $\rho(x)=d_{N}(x, o)$ is the distance function on $N$, then ([GW], Example 2.25)

$$
H \rho=\beta \operatorname{coth} \beta \rho\left(g_{N}-d \rho \otimes d \rho\right),
$$

so if $\left\{Y_{1}=\nabla \rho, Y_{2}, \ldots, Y_{n}\right\}$ is an orthonormal frame on $N$, and $\mu_{i}=H \rho\left(Y_{i}, Y_{i}\right)$, then

$$
\mu_{1}=0 \quad \text { and } \quad \mu_{i}=\beta \operatorname{coth} \beta \rho \quad \text { for } \quad i \geq 2 .
$$

Let $x \in M$ be a point $r=d\left(x, x_{0}\right)$ from the pole, such that $r>\epsilon$. Choose $y \in N$ such that $r=d_{N}(y, o)$. Then the Hessian comparison theorem for manifolds with pole ([GW], Theorem A) shows that

$$
\max _{i=2, \ldots, n} \lambda_{i} \leq \min _{i=2, \ldots, n} \mu_{i}=\beta \operatorname{coth} \beta r=O(1)
$$

since $K_{M} \geq K_{N}$.

Similarly, since $K_{M} \leq K_{R^{n}}=0$, we have

$$
\min _{i=2, \ldots, n} \lambda_{i} \geq \frac{1}{r} .
$$

\section{Every Topologically Tame Manifold has a Strong Hodge Decomposition}

In this section we will choose both a metric $g$ and a measure $d \mu$ on a smooth manifold-under a topological restriction-and show that the resulting $\triangle_{\mu}^{U}$ has compact resolvent. By this construction, we conclude that "every topologically tame smooth manifold has a strong Hodge decomposition," for a suitable Riemannian metric and a suitable measure.

10.1 Definition. Let $M$ be a connected $C^{\infty}$ manifold without boundary. We say $M$ is topologically tame if there exists a compact manifold $N$ with boundary such that $M$ is diffeomorphic to the interior of $N$.

Note that both compact manifolds and vector bundles over compact base manifolds are topologically tame. 
10.2 Theorem. Let $M^{n}$ be a $C^{\infty}$, orientable, connected, and topologically tame manifold. There exists a metric $g$ and a finite measure $d \mu$ with smooth density with respect to $d x$ such that

(i) $\left(M^{n}, g\right)$ is complete Riemannian, and

(ii) $\triangle_{\mu}^{U}$ in $L^{2} \Omega^{\bullet}$ has compact resolvent.

Under the metric $g$, the ends of $M$ are flat cylinders near infinity. The density of the chosen measure $d \mu$ behaves precisely as $\exp \left(c r^{2}\right)$ near infinity on the ends, for any chosen $c \neq 0$.

Proof. We may take $M$ to be the interior of a compact manifold (with boundary) $N$. Let $T$ be a neighborhood of $\partial N$ in $N$ such that

$$
T \cong(0,+\infty] \times \partial N \text {. }
$$

Since $\partial N$ is compact, the interior part of $T$ can be decomposed into a finite list of disjoint open "ends":

$$
T \backslash\{+\infty \times \partial N\}=\bigsqcup_{j=1}^{k} E_{j}
$$

where for each $j$ there exists a diffeomorphism

$$
\varphi_{j}: E_{j} \rightarrow(0,+\infty) \times Q_{j}, \quad Q_{j}^{n-1} \text { compact }, \quad \partial Q_{j}=\emptyset .
$$

Let $\xi_{0}: M \rightarrow[0,1]$ be a $C^{\infty}$ function with compact support such that $\left.\xi_{0}\right|_{M \backslash\left(\cup E_{j}\right)}$ $\equiv 1$. Let

$$
\xi_{j}=\left(1-\xi_{0}\right) \cdot \chi_{E_{j}}
$$

so $\xi_{j}$ is $C^{\infty}$ and supported in $E_{j}$, and $\sum_{j=0}^{k} \xi_{j} \equiv 1$ on $M$.

Choose any Riemannian metric $g_{0}$ on $M$ and any $g_{j}$ on $Q_{j}$. Taking the standard metric $g_{R}$ on $(0,+\infty)$, let

$$
g=\xi_{0} g_{0}+\sum_{j=1}^{k} \xi_{j} \varphi_{j}^{*}\left(g_{R} \oplus g_{j}\right) .
$$

Then $(M, g)$ is complete Riemannian, since each end $E_{j}$ of $M$ is a straight cylinder of infinite length, so closed balls of finite radius are contained in easily constructed compact subsets of $M$.

Let $h_{j}(r, z)=\frac{c}{2} r^{2}$ on $(0,+\infty) \times Q_{j}$. Let

$$
h=\sum_{j=1}^{k} \xi_{j}\left(h_{j} \circ \varphi_{j}\right),
$$

so $h$ is $C^{\infty}$ on $M$, and $|h| \rightarrow \infty$ at $\infty$. Let $d \mu=e^{2 h} d x$. Construct $\triangle_{\mu}^{U}=\triangle+V_{\mu}$ in $L^{2} \Omega^{\bullet}$ :

$$
V_{\mu}=|d h|^{2}+\sum_{i=1}^{n} H h\left(X_{i}, X_{j}\right)\left[a^{i}, a^{i *}\right]
$$

Consider a single end $E_{j}$. Recall $\xi_{0}$ had compact support in $M$. Let $\hat{E}_{j}=$ $E_{j} \backslash \operatorname{supp} \xi_{0}$, and write $r$ for $r \circ \varphi_{j}$. Then $g=\varphi_{j}^{*}\left(g_{R} \otimes g_{j}\right)$ and $h=\frac{c}{2} r^{2}$ on $\hat{E}_{j}$. Let $U$ be a coordinate chart on $Q_{j}$, and let $\hat{U}=\varphi_{j}^{-1}((0, \infty) \times U) \cap \hat{E}_{j}$. Let 
$X_{2}, \ldots, X_{n}$ be orthonormal vector fields on $U$, and let $X_{1}=\frac{\partial}{\partial r}$ on $(0, \infty)$. Then $\left\{\left(\varphi_{j}^{-1}\right)_{*}\left(X_{j}\right)\right\}_{k=1}^{n}$ are orthonormal on $\hat{U}$.

Now,

$$
H r=0
$$

on $\hat{U}$, since $\left(\nabla_{X_{k}} X_{l}\right)(r)=0$ on $(0, \infty) \times U$ for $k, l \geq 2$, and

$$
H\left(r \circ \varphi_{j}\right)\left(\left(\varphi_{j}^{-1}\right)_{*}(X),\left(\varphi_{j}^{-1}\right)_{*}(\nabla r)\right)=0
$$

for any $X$ on $(0, \infty) \times U$. Thus

$$
H h=c \varphi_{j}^{*}(d r \otimes d r)
$$

on $\hat{U}$. Since $|\nabla r|=1$,

$$
|\nabla h|=c^{2} r^{2}
$$

on $\hat{E}_{j}$ and

$$
V_{\mu} \xi=c^{2} r^{2} \xi+c\left[a^{1}, a^{1 *}\right] \xi
$$

on $\xi \in \Omega_{c}^{\bullet}$ supported in $\hat{E}_{j}$, where $a^{1 *} \xi=d r \wedge \xi$.

Since the above applies to every end $E_{j}$, we see that $V_{\mu}$ goes to $\infty$ at $\infty$ if $c \neq 0$, so $\triangle_{\mu}^{U}$ has compact resolvent by Theorem 4.5.

The idea of the above proof is to turn each "end" of $M$ into an infinitely long flat cylinder, and then use the "distance along the end" function $r$ to construct the measure $d \mu=\exp \left(c r^{2}\right) d x$ and the associated potential $V$. Note that $r$ is not a true distance function, but it is indistinguishable from one for large $r$. Note also that if $M$ is compact, then the proof reduces to just choosing a metric $g$ and constructing $\triangle$ - for we can choose $\xi_{0} \equiv 1$ on all of $M$.

We confidently

10.3 Conjecture. Let $M^{n}$ be a $C^{\infty}$, orientable, connected and topologically tame manifold. Choose $g$ as in Theorem 10.2, so $\left(M^{n}, g\right)$ is complete and has flat cylindrical ends. Choose $t>0$ and $x_{0} \in M$. Let

$$
d \mu=\rho_{t}\left(x_{0}, x\right) d x .
$$

Then

$$
\triangle_{\mu}^{U} \text { in } L^{2} \Omega^{\bullet} \text { has compact resolvent. }
$$

\section{REFERENCES}

[A] Michael T. Anderson, $L^{2}$ harmonic forms on complete Riemannian manifolds, in "Geometry and Analysis on Manifolds: Proceedings Katata-Kyoto 1987," (Toshikazu Sunada, ed.) Springer, Berlin (1988), 1-19. MR 89j:58004

[AV] A. Andreotti and E. Vesentini, Carleman estimates for the Laplace-Beltrami equation on complex manifolds, Publications mathematiques de l'Institut der Hautes Etudes Scientifiques no. 25 (1965). MR 30:5333

[AM] A. Arai \& I. Mitoma, De Rham-Hodge-Kodaira decomposition in $\infty$-dimensions, Math. Ann. 291 (1991), 51-73. MR 92k:58007

[Bi] Jean-Michel Bismut, The Witten complex and the degenerate Morse inequalities, J. Differential Geom. 23 (1986), 207-240. MR 87m:58169

[Cha1] Issac Chavel, Eigenvalues in Riemannian Geometry, Academic Press, Orlando, 1984. MR 86g:58140

[Cha2] Issac Chavel, Riemannian Geometry-A Modern Introduction, Cambridge University Press, Cambridge, U. K., 1993. MR 95j:53001 
[CG] J. Cheeger \& D. Gromoll, On the structure of complete manifolds of nonnegative curvature, Ann. of Math. 96 (1972), 413-443. MR 46:8121

[Che] Paul R. Chernoff, Essential self-adjointness of powers of generators of hyperbolic equations, J. Funct. Anal. 12 (1973), 401-414. MR 51:6119

[CFKS] Hans L. Cycon, Werner Kirsch, Richard G. Froese, \& Barry Simon, Schrödinger Operators with Application to Quantum Mechanics and Global Geometry, Springer, Berlin, 1987. MR 88g:35003

[Da1] E. B. Davies, Heat Kernels and Spectral Theory, Cambridge University Press, Cambridge, U. K., 1989. MR 90e:35123

[Da2] E. B. Davies, Pointwise bounds on the space and time derivatives of heat kernels, J. Operator Theory 21 (1989), 367-378. MR 90k:58214

[Dod] Jozef Dodziuk, $L^{2}$-harmonic forms on rotationally symmetric Riemannian manifolds, Proc. Amer. Math. Soc. 77 no. 3 (1979), 395-400. MR 81e:58004

[Don] Harold Donnelly, The differential form spectrum of hyperbolic space, Manuscripta Math. 33 (1981), 365-385. MR 82f:58085

[DonL] Harold Donnelly \& Peter Li, Lower Bounds for the Eigenvalues of Riemannian Manifolds, Michigan Math. J. 29 (1982), 149-161. MR 83g:58069

[DonX] Harold Donnelly \& Frederico Xavier, On the differential form spectrum of negatively curved Riemannian manifolds, Amer. J. Math. 106 (1984), 169-185. MR 85i:58115

[DriH] Bruce Driver \& Yaozhong Hu, On heat kernel logarithmic Sobolev inequalities, in "Proceedings of the Fifth Gregynog Symposium" (I. M. Davies et al. eds.) Stochastic Analysis and Applications, World Scientific, NJ (1996), 189-200. MR 98h:58183

[DriL] Bruce Driver \& Terry Lohrenz, Logarithmic Sobolev inequalities for pinned loop groups, J. Funct. Anal. 40 (1996), 381-448. MR 97h:58176

[ER] K. D. Elworthy \& S. Rosenberg, The Witten Laplacian on negatively curved simply connected manifolds, Tokyo J. Math. 16 (1992), 513-524. MR 94j:58171

[EF1] Jose F. Escobar \& Alexandre Freire, The spectrum of the Laplacian of manifolds of positive curvature, Duke Math. J. 65, no. 1 (1992), 1-21. MR 93d:58174

[EF2] Jose F. Escobar \& Alexandre Freire, The differential form spectrum of manifolds of positive curvature, Duke Math. J. 69, no. 1 (1993), 1-41. MR 94b:58097

[Ga] Matthew P. Gaffney, Hilbert space methods in the theory of harmonic integrals, Trans. Amer. Math. Soc. 78 (1955), 426-444. MR 16:957a

[GW] R. E. Greene \& H. Wu, Function Theory on Manifolds Which Possess a Pole, Lecture Notes in Mathematics 699, Springer, Berlin, 1979. MR 81a:53002

[Gri] A. A. Grigor'yan, Heat kernel of a noncompact Riemannian manifold, Stochastic analysis (Ithaca, NY, 1993), Proc. Sympos. Pure Math., Amer. Math. Soc., Providence, RI 57 (1995), 239-263. MR 96f:58155

[Gro] Leonard Gross, Analysis on Loop Groups, in "Stochastic Analysis and Applications in Physics" (A.I. Cardoso, et al., eds.) Kluwer Academic Publishers, Dordrecht (1994). MR 96j:58023

[Hs] Elton P. Hsu, Logarithmic Sobolev Inequalities on Path Space, C. R. Acad. Sci. Paris Sér. I Math. 320 (1995), 1009-1012. MR 96e:58167

[K1] S. Kusuoka, Analysis on Wiener spaces I. Nonlinear maps, J. Funct. Anal. 98 (1991), 122-168. MR 93a:58176

[K2] S. Kusuoka, Analysis on Wiener spaces II. Differential forms, J. Funct. Anal. 103 (1992), 229-274. MR 93c:58230

[K3] S. Kusuoka, De Rham cohomology of Wiener-Riemannian manifolds, in "Proceedings of the International Congress of Mathematicians (Kyoto, 1990)," Math. Soc. Japan, Tokyo (1991), 1075-1082. MR 93e:58198

[L] John Lott, The Zero-in-the-Spectrum Question, Enseign. Math. (2) 42 (1996), 341-376. MR 97h:58145

[MS] P. Malliavin \& D. Stroock, Short time behavior of the heat kernel and its logarithmic derivatives, J. Differential Geomerty 44 (1996), 550-570. MR 98c:58164

[P] M. Ann Piech, The Exterior Algebra of Wiemann Manifolds, J. Funct. Anal. 28 (1978), 279-308. MR 58:2851

[RaS] D. B. Ray \& I. M. Singer, R-Torsion and the Laplacian on Riemannian Manifolds, Adv. Math. 7 (1971), 145-210. MR 45:4447 
[ReS] Michael Reed \& Barry Simon, Methods of Modern Mathematical Physics, Volume II: Fourier Analysis, Self-Adjointness, Academic Press, New York, 1975. MR 58:12429b

[deR] Georges de Rham, Differentiable Manifolds, Springer, Berlin, 1984. MR 85m:58005

[Sh] Ichiro Shigekawa, De Rham-Hodge-Kodaira's decomposition on an abstract Wiener space, J. Math. Kyoto Univ. 26 (1986), 191-202. MR 88h:58009

[St] Daniel W. Stroock, An Estimate on the Hessian of the Heat Kernel, in "Itô's Stochastic Calculus and Probability Theory" (N. Ikeda et al. eds.), Springer, Tokyo (1996). MR 97m:58212

[Wa] Frank W. Warner, Foundations of Differentiable Manifolds and Lie Groups, Springer, New York, 1983. MR 84k:58001

[Wi] Edward Witten, Supersymmetry and Morse theory, J. Differential Geom. 17 (1982), 661692. MR 84b:58111

Department of Mathematics Sciences, University of Alaska, Fairbanks, Alaska 99775 